\title{
Bifurcation Investigations of Coupled Damage-Plasticity Models for Concrete Materials
}

\author{
G. Xotta ${ }^{\mathrm{a}, \mathrm{b}, *}$, S. Beizaee ${ }^{\mathrm{a}}$, K.J. Willam ${ }^{\mathrm{a}}$ \\ ${ }^{a}$ Department of Civil and Environmental Engineering, Cullen College of Engineering, \\ University of Houston, N107 Engineering Building 1, Houston TX 77204-4003, USA. \\ E-mail addresses: sbeizaee@uh.edu; kwillam@uh.edu \\ ${ }^{b}$ Department of Civil, Environmental and Architectural Engineering (DICEA), \\ University of Padova, Via F. Marzolo 9, 35131 Padova, Italy.E-mail address: \\ giovanna.xotta@dicea.unipd.it
}

\section{Abstract}

This communication addresses the localization properties of a coupled damageplasticity formulation for concrete materials to provide informations on the onset of material bifurcation and the critical failure modes.

Two separate loading functions are considered, one for damage and one for plasticity. A three-invariant yield surface is used to model plasticity and to consider the significant role of the intermediate principal stress and the Lode parameter on the failure of concrete materials. A non-associated flow rule is employed to control inelastic dilatancy. To model degradation of the elastic stiffness a scalar-valued isotropic damage formulation is introduced based on the total strain energy formulation is used.

Monotonic and cyclic uniaxial compression experiments are performed on concrete cylinders under displacement control and photogrammetric images are collected for Digital Image Correlation Analysis. The triaxial based

\footnotetext{
${ }^{*}$ Corresponding Author, Tel.:+390498275592
}

Preprint submitted to Computational Methods in Applied Mechanics and EngineeringAugust 8, 2015 
damage-plasticity model is calibrated based on these experimental observations and is implemented in Matlab.

Extensive localization analysis studies are performed at the constitutive level for representative load scenarios in the form of non-positive properties of the elastoplastic-damage localization tensor. The contributions of damage, plasticity and coupled damage-plasticity are explored and compared for classical Boltzmann and Micropolar Cosserat continuum formulations.

Keywords: Concrete Materials, Localization, Coupling of three-invariant plasticity and damage

\section{Introduction}

A survey of recent concrete literature indicates a rapidly increasing number of proposals to combine plasticity and continuum damage mechanics for the characterization of concrete materials (Lee and Fenves [1], Carol et al [2], Salari et al. [3], Tao and Phillips [4], Grassl and Jirásek, [5,6]; Cérvenka and Papanikolaou [7], Voyiadjis et al. [8], Grassl et al. [9)]. Thereby, it is understood that both damage and plasticity formulations are dissipative in nature, even though they start from very different constitutive concepts. Irrespective of the thermodynamic setting, continuum damage mechanics resorts to the reduction of the initial elastic stiffness properties in form of a Cauchy type elastic secant relationship. In contrast plasticity resorts to the notion of strength and its description of an evolving yield surface.

In the case of scalar damage the entire stiffness tensor is reduced by the scalar valued (1-D) factor, which retains the fundamental construct of linear elasticity (isotropic or anisotropic). Consequently, the main deficiency 
of continuum damage mechanics is the initial reference stiffness, which does not change when the concrete exhibits strongly dilatant behavior e.g. in compression when the stress reaches the maximum material resistance. The so-called Reynolds effect of coupling shear and volume change is a classical attribute of granular materials. Therefore, traditional concrete exhibits a transition from compaction to dilatation under increasing axial compression. This transition of volume change is missing in scalar-valued damage models when Poisson's ratio remains fixed (this deficiency cannot be remedied by an isotropic damage formulation based on one or two separate damage variables characterizing volumetric and deviatoric damage processes with no coupling). The other deficiency of continuum damage models is the reversibility of deformations under load-unload cycles, which does not account for permanent deformations.

In the case of elasto-plasticity, it is the question of the flow rule and hence the plastic potential whether the elastic reference stiffness is sufficiently modified by plastic dilatancy controlled by the volumetric portion of the plastic strain rate of the flow rule. In fact, most pressure-sensitive plasticity formulations adopt a non-associated flow rule to reduce the plastic dilatation in granular media and concrete. Clearly, the main attribute of plasticity is the irreversible nature of the plastic deformations under load cycles. Therefore, the main deficiencies of continuum damage models may be corrected by the combination of the two dissipative mechanisms of elastic damage and plasticity.

In this paper a damage-plasticity formulation is adopted to characterize concrete's behaviour, where damage and plasticity functions are two separate 
functions, as discussed by Grassl and Jirásek [5, 6]. In specific, for platicity, the three-invariant yield surface of Willam-Warnke [10] is chosen, in order to consider the important role of the intermediate principal stress in concrete materials and a non-associated flow rule is employed to control inelastic dilatancy. To model damage behavior, the formulation based on total strain energy is adopted, in a format presented by Salari et al. [3] .

Complex response phenomena of concrete materials (inelastic dilatancy, pressure sensitivity etc..) are at the basis of specific failure characteristics and the formation of cracks and shear bands that are typical examples of localized failure mechanisms. Localization analysis in form of bifurcation studies of the localization tensor signal the formation of weak $C^{-1}$ discontinuities in the velocity field synonymous with the loss of ellipticity.

Original works in this field are to be attributed to Rudnicki and Rice [11] and Rice and Rudnicki [12] for pressure-sensitive plasticity, based on original theories by Hadamard [13] and Hill [14] regarding bifurcation on stationary acceleration waves.

A number of authors investigated the bifurcation problem and developed algorithms for a variety of elasto-plastic concrete models, extending, in some cases, the results when considering also the presence of damage (Bigoni and Hueckel[15,16], Kang and Willam[17], Liebe and Willam [18], Rizzi et al. [19], Steinmann and Willam [20], Vrech and Etse [21], Willam and Iordache $[22,23])$.

Cosserat or micropolar continuum formulations [24] were introduced to incorporate gradients and length scales of the material microstructure within a consistent framework of continuous media. The general formulation was 
first introduced by Cosserat and Cosserat [25, 26]. The elastoplastic Cosserat formulation was described by Willam et al. [27] and De Borst [28] and the localization analysis of this higher order continua was extensively discussed by Willam et al. [27]. Detailed information about Cosserat implementation and formulation for finite element analysis was presented in papers by Kondo [29].

\section{Theoretical Framework}

In this section, the governing equations for the Boltzmann and Cosserat continua are presented. The theoretical background for elastoplastic material behavior and damage-plasticity formulation is delineated. Menétrey-Willam [31] plasticity and damage, as a separate loading function, criteria are introduced and the coupling of damage and plasticity based on effective stresses is described.

\subsection{Boltzmann Continuum}

In order to characterize the motion of a body, the equilibrium, the kinematic and the constitutive equations are considered.

The strong format of the equilibrium equation for a static problem may be expressed as follows:

$$
\nabla \cdot \boldsymbol{\sigma}+\rho \mathbf{b}=0
$$

where $\rho \cdot \mathbf{b}$ is a vector of body forces.

The kinematic equations based on infinitesimal strain theory are defined as:

$$
\boldsymbol{\epsilon}=\nabla_{\text {sym }} \cdot \mathbf{u}
$$


and the following expression for linear elasticity:

$$
\boldsymbol{\sigma}=\mathbf{E}^{e}: \epsilon
$$

The stress and strain tensors, seen in the previous equations, for 2D material behavior are so defined:

$$
\boldsymbol{\sigma}=\left[\sigma_{11} \sigma_{22} \sigma_{33} \sigma_{12}\right]^{T} \text { and } \boldsymbol{\epsilon}=\left[\epsilon_{11} \epsilon_{22} \epsilon_{33} \epsilon_{12}\right]^{T} .
$$

It should be noted that Voigt notation is used to represent the stress and strain tensors which are symmetric in the non-polar case when $\sigma_{12}=\sigma_{21}$ and $\epsilon_{12}=\epsilon_{21}$.

The linear elastic stiffness tensor may be expressed in matrix form as:

$$
\mathbf{E}^{e}=\frac{E}{(1+\nu)(1-2 \nu)}\left[\begin{array}{cccc}
1-\nu & \nu & \nu & 0 \\
\nu & 1-\nu & \nu & 0 \\
\nu & \nu & 1-\nu & 0 \\
0 & 0 & 0 & \frac{(1-2 \nu)}{2}
\end{array}\right]
$$

where $E$ is the Young's modulus and $\nu$ is the Poisson's ratio.

In order to further extend the formulations for plasticity, the invariants of stresses are introduced:

$$
\begin{aligned}
& I_{1}=\operatorname{tr}(\boldsymbol{\sigma})=\sigma_{11}+\sigma_{22}+\sigma_{33}, \\
& J_{2}=\frac{1}{2} \operatorname{tr}\left(\mathbf{s}^{2}\right)=\frac{1}{2}\left(s_{i j} s_{i j}\right), \\
& J_{3}=\frac{1}{3} \operatorname{tr}\left(\mathbf{s}^{3}\right)=\frac{1}{3}\left(s_{i j} s_{j k} s_{k i}\right)
\end{aligned}
$$

where $\mathbf{s}$ is the deviatoric stress tensor and is defined by subtracting the sum of hydrostatic stresses, as here reported:

$$
\mathbf{s}=\boldsymbol{\sigma}-\frac{1}{3} I_{1} \mathbf{1}
$$

and $\mathbf{1}$ is the identity tensor. 


\subsection{Cosserat Continuum}

The equilibrium equations for Cosserat continuum, in absence of body forces and body couples, are described as:

$$
\nabla \cdot \boldsymbol{\sigma}=0 \quad \text { and } \quad \nabla \cdot \mathbf{m}+\mathbf{e}: \boldsymbol{\sigma}=0
$$

where $\mathbf{m}$ represents the couple stresses or moments and $\mathbf{e}$ is the permutation tensor. The stress representation for a 2D Cosserat or micropolar continuum element is depicted in Figure 1.

The kinematic equations for micropolar continua encompass the equations

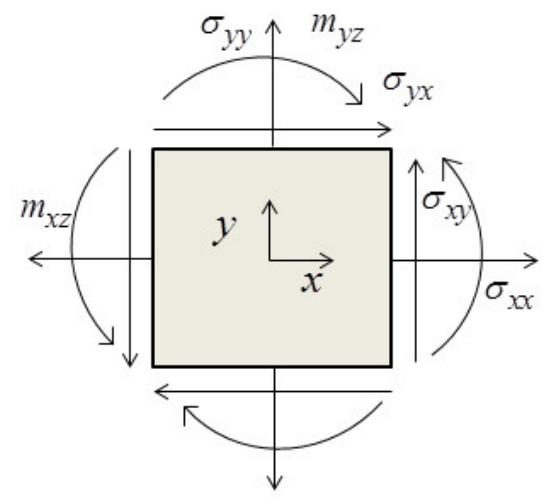

Figure 1: Stress representation of Cosserat continua in 2D.

for displacements and the equations for the micro-rotations and are so formulated:

$$
\boldsymbol{\epsilon}=\nabla \mathbf{u}-\mathbf{e} \cdot \boldsymbol{\omega} \quad \text { and } \quad \boldsymbol{\kappa}=\nabla \boldsymbol{\omega}
$$

where $\boldsymbol{\kappa}$ is the micro-curvature, that is energetically conjugated to couple stresses. The micro-rotations are defined by $\boldsymbol{\omega}$.

The kinematics of macro and micro rotations are depicted in Figure 2. 


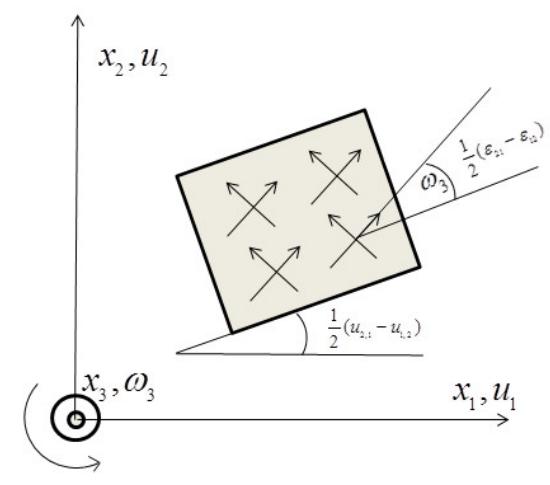

Figure 2: Macro and micro-rotations in Cosserat continua.

In order to simplify the numerical implementation of the Cosserat continuum, it is assumed that the stress and strain tensors include the couple stresses $\left(m_{i j}\right)$ and the curvatures $\left(\kappa_{i j}\right)$. Therefore the Voigt notations of these tensors are:

$$
\begin{aligned}
\boldsymbol{\sigma} & =\left[\begin{array}{lllllll}
\sigma_{11} & \sigma_{22} & \sigma_{33} & \sigma_{12} & \sigma_{21} & \frac{m_{13}}{l} & \frac{m_{23}}{l}
\end{array}\right]^{T}, \\
\boldsymbol{\epsilon} & =\left[\begin{array}{llllllll}
\epsilon_{11} & \epsilon_{22} & \epsilon_{33} & \epsilon_{12} & \epsilon_{21} & \kappa_{13} l & \kappa_{23} l
\end{array}\right]^{T}
\end{aligned}
$$

where $l$ is the length factor, that is included in the stress and strain tensors to unify the dimensions of these tensors.

The elastic stiffness matrix for Cosserat continuum is expressed in equation (11). Here it is assumed that there is no coupling between the stresses and couple stresses, or strains and curvatures.

It should be mentioned that $\mu$ is the Lamé's second parameter or shear 
modulus, instead the shear parameter $\mu_{c}$ is the Cosserat shear modulus.

$$
\mathbf{E}^{e}=\left[\begin{array}{ccccccc}
2 \mu c_{1} & 2 \mu c_{2} & 2 \mu c_{2} & 0 & 0 & 0 & 0 \\
2 \mu c_{2} & 2 \mu c_{1} & 2 \mu c_{2} & 0 & 0 & 0 & 0 \\
2 \mu c_{2} & 2 \mu c_{2} & 2 \mu c_{1} & 0 & 0 & 0 & 0 \\
0 & 0 & 0 & \mu+\mu_{c} & \mu-\mu_{c} & 0 & 0 \\
0 & 0 & 0 & \mu-\mu_{c} & \mu+\mu_{c} & 0 & 0 \\
0 & 0 & 0 & 0 & 0 & 2 \mu & 0 \\
0 & 0 & 0 & 0 & 0 & 0 & 2 \mu
\end{array}\right] ; \quad \begin{gathered}
c_{1}=\frac{1-\nu}{1-2 \nu} . \\
c_{2}=\frac{\nu}{1-2 \nu}
\end{gathered}
$$

In order to further explore the Cosserat formulation for plasticity, it is necessary to define the stress invariants as follows (see the expanded format in the APPENDIX):

$$
\begin{aligned}
& I_{1}=\operatorname{tr}(\boldsymbol{\sigma})=\sigma_{11}+\sigma_{22}+\sigma_{33}, \\
& J_{2}=\frac{1}{2}\left(s_{i j} s_{i j}+\frac{1}{l^{2}} m_{i j} m_{i j}\right), \\
& J_{3}=\frac{1}{3} \operatorname{tr}\left(\mathbf{s}^{3}\right)=\frac{1}{3}\left(s_{i j} s_{j k} s_{k i}+\frac{1}{f\left(l, l_{t}\right)} m_{i j} m_{j k} m_{k i}\right) .
\end{aligned}
$$

where $l_{t}$ is the torsional length scale.

It should be mentioned that, for calculating the elastoplastic tangent operator, it is possible to decompose the stiffness matrix as follows:

$$
\mathbf{E}=\left[\begin{array}{cc}
\mathbf{E}^{u u} & 0 \\
0 & \mathbf{E}^{\omega \omega}
\end{array}\right]
$$

where superscripts $u$ and $\omega$ represent, respectively, the Boltzmann and Cosserat continua. 


\subsection{Elasto Plastic Material Behaviour}

As the material reaches its proportional or yield limit, it undergoes permanent deformations, i.e. in the case of unloading the stress-strain curve does not return to its original place. This behavior can be explained by plasticity theory. In this work it is assumed that the plastic yield function is calculated based on effective stresses and consequenlty there is no direct coupling of the damage variable in the plastic part.

In this work the Menétrey and Willam [31] format of the Willam-Warnke [10] yield surface is adopted (see Figure 3).

This yield criterion can be expressed in terms of the Haigh-Westergaard co-
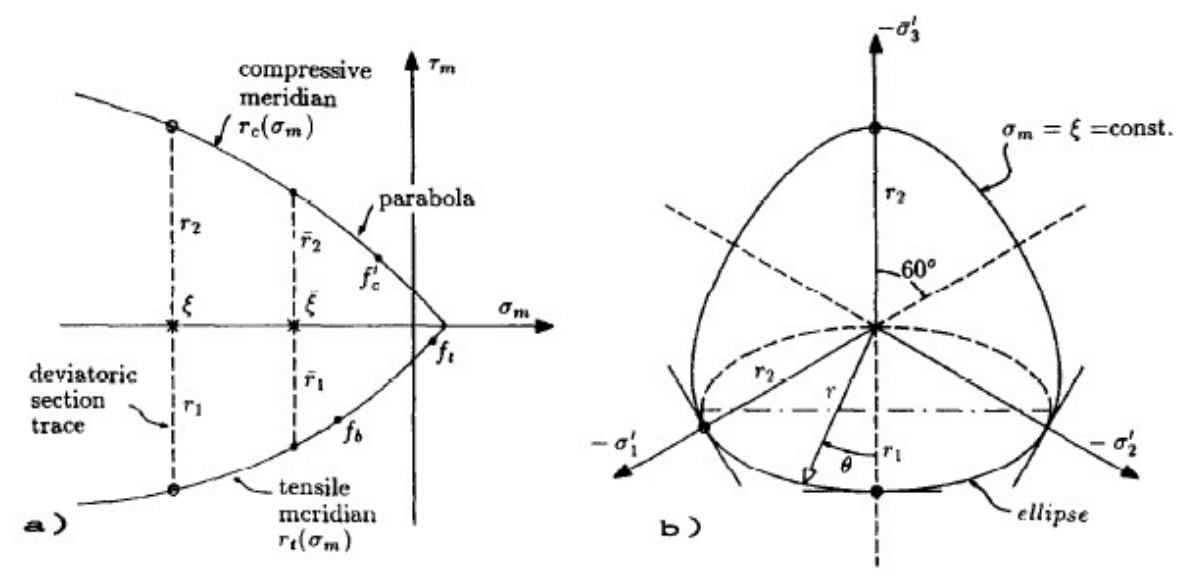

Figure 3: Five-parameter model (Willam and Warnke [10]): a)hydrostatic section; b) deviatoric section.

ordinates (the bar sign indicates that these coordinates are calculated based on effective stresses, $\overline{\boldsymbol{\sigma}}$, and not based on the nominal ones) as follows:

$$
f^{p}(\bar{\xi}, \bar{\rho}, \bar{\theta}, k)=\left(\frac{\sqrt{1.5}}{f_{c}} \bar{\rho}\right)^{2}+q_{h} m\left[\frac{\bar{\rho}}{\sqrt{6} f_{c}} r(\bar{\theta}, e)+\frac{\bar{\xi}}{\sqrt{3} f_{c}}\right]-q_{h} q_{s}=0
$$


where $q_{h}$ and $q_{s}$ are respectively the hardening and the softening functions, which depend on the internal variable $k$ (here equal to the effective plastic strain $\left.\epsilon_{e q}^{p}\right), m$ is the cohesion parameter of the material, $r$ is a function of $\bar{\theta}$ and $e$ that defines the roundness of the failure surface. Please refer to Menétrey and Willam [31] and Cěrvenka and Papanikolaou [7] for the terms expressions.

In order to express the rate of plastic strain it is assumed that there exists a potential function $g^{p}$, so expressed:

$$
g^{p}=-A\left(\frac{\bar{\rho}}{\sqrt{q_{h} q_{s}} f_{c}}\right)^{2}-B \frac{\bar{\rho}}{\sqrt{q_{h} q_{s}} f_{c}}+\frac{\bar{\xi}}{\sqrt{q_{h} q_{s}} f_{c}}
$$

where the parameters $A$ and $B$ are obtained by calibrating the model with experimental data.

The plastic flow and the evolution of the internal variables are so defined:

$$
\begin{array}{rlrl}
\dot{\boldsymbol{\epsilon}}^{p} & =\dot{\lambda} \frac{\partial g^{p}}{\partial \overline{\boldsymbol{\sigma}}}=\dot{\lambda} \mathbf{m} & & \dot{\lambda} \geq 0, \\
\dot{k}=\dot{\epsilon}_{e q}^{p}=\dot{\lambda}\left\|\frac{\partial g^{p}}{\partial \overline{\boldsymbol{\sigma}}}\right\|=\dot{\lambda}\|\mathbf{m}\| & \dot{\lambda} \geq 0
\end{array}
$$

where $\mathbf{m}$ is the normal to the potential function. The flow rule states that the direction of the plastic strain is expressed by $\mathbf{m}$ and the plastic multiplier $\dot{\lambda}$ determines the magnitude of $\dot{\boldsymbol{\epsilon}}^{p}$.

In order to have plastic loading the plastic multiplier has to be equal or greater than zero while the yield function has to be equal or less than zero and the product of these two has to remain zero at all instances. This is expressed as:

$$
\dot{\lambda} \geq 0, \quad f^{p}(\overline{\boldsymbol{\sigma}}, \mathbf{q}) \leq 0, \quad \dot{\lambda} \cdot f^{p}(\overline{\boldsymbol{\sigma}}, \mathbf{q})=0 .
$$

In order to have a thermodynamically admissible solution, the dissipation or Clausius-Duhem inequality should be satisfied, for the hardening/softening 
variables selected:

$$
\boldsymbol{\sigma}: \dot{\boldsymbol{\epsilon}}^{p}-\mathbf{q} \cdot \dot{\mathbf{k}} \geq 0 .
$$

Established the yield function and the flow rules, the Prager's consistency condition is introduced in equation (19), stating that, during the development of plastic strain, the yield criterion should be fulfilled:

$$
\dot{f}^{p}=\frac{\partial f^{p}}{\partial \overline{\boldsymbol{\sigma}}}: \dot{\overline{\boldsymbol{\sigma}}}+\frac{\partial f^{p}}{\partial \epsilon_{e q}^{p}}: \dot{\epsilon}_{e q}^{\dot{p}}=0 .
$$

Once adopted the following notations to simplify the expression:

$$
\mathbf{n}=\frac{\partial f^{p}}{\partial \overline{\boldsymbol{\sigma}}} \quad \text { and } \quad l=\frac{\partial f^{p}}{\partial \epsilon_{e q}^{p}}
$$

equation (19) is exploited in order to find the plastic multiplier:

$$
\mathbf{n}: \mathbf{E}^{e}: \dot{\boldsymbol{\epsilon}}-\dot{\lambda} \mathbf{n}: \mathbf{E}^{e}: \mathbf{m}+\dot{\lambda} l\|\mathbf{m}\|=0 \Rightarrow \dot{\lambda}=\frac{\mathbf{n}: \mathbf{E}^{e}: \dot{\boldsymbol{\epsilon}}}{\mathbf{n}: \mathbf{E}^{e}: \mathbf{m}-l\|\mathbf{m}\|}
$$

Afterwards this plastic multiplier is substituted into the rate equation:

$$
\dot{\bar{\sigma}}=\mathbf{E}^{e}: \dot{\boldsymbol{\epsilon}}-\dot{\lambda} \mathbf{E}^{e}: \mathbf{m}
$$

to form the elastoplastic continuum tangent operator:

$$
\mathbf{E}^{e p}=\mathbf{E}^{e}-\frac{1}{H^{p}} \mathbf{E}^{e}: \mathbf{m} \otimes \mathbf{n}: \mathbf{E}^{e}
$$

where the hardening parameter in the denominator is defined as

$$
H^{p}=\mathbf{n}: \mathbf{E}^{e}: \mathbf{m}-l\|\mathbf{m}\| .
$$

In the case of Cosserat continuum it is assumed that there is no coupling between the macro and micro behaviors. To find the tangent operator it has to be taken into account that the elastic stiffness matrix is composed of four 
parts and consequently also the tangent operator is decomposed into four matrices:

$$
\mathbf{E}^{e p}=\left[\begin{array}{cc}
\mathbf{E}_{u u}^{e p} & 0 \\
0 & \mathbf{E}_{\omega \omega}^{e p}
\end{array}\right]
$$

where these components explicitly assume the following expressions:

$$
\begin{gathered}
\mathbf{E}_{u u}^{e p}=\mathbf{E}_{u u}^{e}-\frac{1}{H_{c}^{p}} \mathbf{E}_{u u}^{e}: \mathbf{m}^{u} \otimes \mathbf{n}^{u}: \mathbf{E}_{u u}^{e} \\
\mathbf{E}_{\omega \omega}^{e p}=\mathbf{E}_{\omega \omega}^{e}-\frac{1}{H_{c}^{p}} \mathbf{E}_{\omega \omega}^{e}: \mathbf{m}^{\omega} \otimes \mathbf{n}^{\omega}: \mathbf{E}_{\omega \omega}^{e}
\end{gathered}
$$

The hardening parameter in the denominator of equations (26) is defined as

$$
H_{c}^{p}=\mathbf{n}^{u}: \mathbf{E}_{u u}^{e}: \mathbf{m}^{u}+\mathbf{n}^{\omega}: \mathbf{E}_{\omega \omega}^{e}: \mathbf{m}^{\omega}-l\|\mathbf{m}\|
$$

where the superscripts of $\mathbf{m}$ and $\mathbf{n}$ define whether these vectors are for displacement $(u)$ or rotation $(\omega)$ components.

\subsection{Damage Formulation}

The damage formulation is based on the total elastic energy and the accumulated plastic strain energy. The damage function consists of two parts, the damage energy and the resistance function, and assumes the following expression:

$$
f^{d}=f^{d}\left(\boldsymbol{\epsilon}^{e}, \boldsymbol{\epsilon}^{p}, D\right)=Y-r
$$

where $Y$ is the damage energy:

$$
Y=p_{D} \frac{1}{2} \boldsymbol{\epsilon}^{e}: \mathbf{E}^{e}: \boldsymbol{\epsilon}^{e}+c \int \boldsymbol{\sigma}: d \boldsymbol{\epsilon}^{p}
$$

while the resistance function $r$ is defined as:

$$
r=r_{0}(1-D)^{p-1}
$$


where $r_{0}$ is the modulus of resilience and $p$ represents the ratio of modulus of resilience and modulus of toughness (see [3]). $p_{D}$ and $c$ terms, present in equation (29), have different values for different loading conditions.

The rate of the damage function, expressed in terms of the plastic multiplier and the damage variable, becomes:

$\dot{f}^{d}=p_{D} \boldsymbol{\epsilon}^{e}: \mathbf{E}^{e}: \dot{\boldsymbol{\epsilon}}-\dot{\lambda}\left(p_{D} \boldsymbol{\epsilon}^{e}: \mathbf{E}^{e}: \dot{\mathbf{m}}-c \boldsymbol{\sigma}: \mathbf{m}\right)+\dot{D}\left[r_{0}(p-1)(1-D)^{p-2}\right]=0$

while the rate of damage is equal to:

$$
\Delta D=\frac{-p_{D} \boldsymbol{\epsilon}^{e}: \mathbf{E}^{e}: \Delta \boldsymbol{\epsilon}+\dot{\lambda}\left(p_{D} \boldsymbol{\epsilon}^{e}: \mathbf{E}^{e}: \dot{\mathbf{m}}-c \boldsymbol{\sigma}: \mathbf{m}\right)}{r_{0}(p-1)(1-D)^{p-2}}
$$

(in the case that plasticity is not present equations (31) and (32) can be suitably simplified).

In order to have damage loading, the damage increment has to be greater or equal to zero, the damage function has to remain negative or equal to zero and the product of these two has to remain zero at all instances. This is expressed as:

$$
\dot{D} \geq 0, \quad f^{d}\left(\boldsymbol{\epsilon}^{e}, \boldsymbol{\epsilon}^{p}, D\right) \leq 0, \quad \dot{D} \cdot f^{d}\left(\boldsymbol{\epsilon}^{e}, \boldsymbol{\epsilon}^{p}, D\right)=0 .
$$

In order to have a thermodynamically admissible solution, the dissipation or Clausius-Duhem inequality should be satisfied:

$$
\boldsymbol{\sigma}: \dot{\boldsymbol{\epsilon}}^{p}-Y \cdot \dot{D} \geq 0
$$

Considering the constitutive rate equation:

$$
\boldsymbol{\sigma}=(1-D) \mathbf{E}^{e}: \boldsymbol{\epsilon}^{e} \Rightarrow \dot{\boldsymbol{\sigma}}=-\dot{D} \mathbf{E}^{e}: \boldsymbol{\epsilon}^{e}+(1-D) \mathbf{E}^{e}: \dot{\boldsymbol{\epsilon}}^{e} .
$$


and substituting $\Delta D$ from equation (32) (simplified considering no plasticity), the elasto-damage continuum tangent operator can be so obtained:

$$
\mathbf{E}^{e d}=p_{D} \frac{\mathbf{E}^{e}: \boldsymbol{\epsilon}^{e} \otimes \boldsymbol{\epsilon}^{e}: \mathbf{E}^{e}}{r_{0}(p-1)(1-D)^{p-2}}+(1-D) \mathbf{E}^{e}
$$

In the case of Cosserat continuum, similarly to the elastoplastic tangent operator, the elasto-damage tangent operator has four components.

In this work it is assumed that damage is only applied to the classical part of the elastic tensor (no damage for couple stresses). Accordingly the rate equations have the following format:

$$
\left[\begin{array}{l}
\boldsymbol{\sigma} \\
\underline{\mu}
\end{array}\right]=\left[\begin{array}{c}
(1-D) \overline{\boldsymbol{\sigma}} \\
\frac{\boldsymbol{\mu}}{l}
\end{array}\right]=\left[\begin{array}{cc}
(1-D) \mathbf{E}_{u u}^{e} & 0 \\
0 & \boldsymbol{E}_{\omega \omega}^{e}
\end{array}\right]\left[\begin{array}{l}
\boldsymbol{\epsilon}^{e} \\
l \boldsymbol{\kappa}
\end{array}\right] .
$$

where the rate of damage could be defined as

$$
\Delta D=\frac{-p_{D}\left[\begin{array}{l}
\boldsymbol{\epsilon}^{e} \\
l \boldsymbol{\kappa}
\end{array}\right]:\left[\begin{array}{cc}
\mathbf{E}_{u u}^{e} & 0 \\
0 & \mathbf{E}_{\omega \omega}^{e}
\end{array}\right]:\left[\begin{array}{l}
\Delta \boldsymbol{\epsilon}^{e} \\
l \Delta \boldsymbol{\kappa}
\end{array}\right]}{r_{0}(p-1)(1-D)^{p-2}}
$$

Substituting the change of damage variable in equation (37), the components of the elasto-damage tangent operator are so obtained:

$$
\begin{aligned}
\mathbf{E}_{u u}^{e d} & =(1-D) \mathbf{E}_{u u}^{e}+\frac{\mathbf{E}_{u u}^{e}: \boldsymbol{\epsilon}^{e} \otimes \boldsymbol{\epsilon}^{e}: \mathbf{E}_{u u}^{e}}{r_{0}(p-1)(1-D)^{p-2}} \\
\mathbf{E}_{u \omega}^{e d} & =\frac{\mathbf{E}_{u u}^{e}: \boldsymbol{\epsilon}^{e} \otimes \boldsymbol{\kappa}^{e}: \mathbf{E}_{\omega \omega}^{e}}{r_{0}(p-1)(1-D)^{p-2}} \\
\mathbf{E}_{\omega u}^{e d} & =\frac{\mathbf{E}_{\omega \omega}^{e}: \boldsymbol{\kappa}^{e} \otimes \boldsymbol{\epsilon}^{e}: \mathbf{E}_{u u}^{e}}{r_{0}(p-1)(1-D)^{p-2}} \\
\mathbf{E}_{\omega \omega}^{e d} & =\mathbf{E}_{\omega \omega}^{e}+\frac{\mathbf{E}_{\omega \omega}^{e}: \boldsymbol{\kappa}^{e} \otimes \boldsymbol{\kappa}^{e}: \mathbf{E}_{\omega \omega}^{e}}{r_{0}(p-1)(1-D)^{p-2}}
\end{aligned}
$$




\subsection{Damage-Plasticity Coupling}

In this section, the damage-plasticity formulation used to model the behavior of concrete is discussed. Menétrey and Willam format of the WillamWarnke yield surface is employed in order to take into account the permanent deformation that retains inside the deteriorated material while a scalar isotropic damage model accounts for the deterioration of the elastic stiffness. Moreover it is assumed that the plasticity and damage have different loading functions.

The Helmholtz free energy function can be additively decomposed into an elastic and a plastic part and may be expressed as:

$$
\psi=\psi^{e}\left(\boldsymbol{\epsilon}^{e}, D\right)+\psi^{p}(k, D)
$$

and therefore the Clausius-Duhem inequality becomes:

$$
\left(\sigma-\frac{\partial \psi^{e}}{\partial \boldsymbol{\epsilon}^{e}}\right): \dot{\boldsymbol{\epsilon}}+\left(\frac{\partial \psi^{e}}{\partial \boldsymbol{\epsilon}^{e}}\right): \dot{\boldsymbol{\epsilon}}^{p}-\left(\frac{\partial \psi^{p}}{\partial k}\right) \cdot \dot{k}-\left(\frac{\partial \psi^{p}}{\partial D}\right) \cdot \dot{D} \geq 0
$$

Considering the fact that the first term of equation (41) is equal to zero and:

$$
\boldsymbol{\sigma}=\frac{\partial \psi^{e}}{\partial \boldsymbol{\epsilon}^{e}}, \quad K=-\left(\frac{\partial \psi^{p}}{\partial k}\right) \quad \text { and } \quad Y=-\left(\frac{\partial \psi^{p}}{\partial D}\right)
$$

the Clausius-Duhem inequality is therefore reformulated as:

$$
\boldsymbol{\sigma}: \dot{\boldsymbol{\epsilon}}^{p}+K \cdot \dot{k}+Y \cdot \dot{D} \geq 0
$$

In order to have a thermodynamically admissible solution, this inequality has to remain positive.

The consistency conditions for the plasticity and damage functions based on 
equations (19) and (31) are so expressed:

$$
\left[\begin{array}{c}
\mathbf{n}: \mathbf{E}^{e}: \mathbf{m}-l\|\mathbf{m}\| \\
p_{D} \boldsymbol{\epsilon}^{e}: \mathbf{E}^{e}: \mathbf{m}-c \boldsymbol{\sigma}: \mathbf{m} \\
-r_{0}(p-1)(1-D)^{p-2}
\end{array}\right]\left[\begin{array}{c}
\dot{\lambda} \\
\dot{D}
\end{array}\right]=\left[\begin{array}{c}
\mathbf{n}: \mathbf{E}^{e}: \dot{\boldsymbol{\epsilon}} \\
p_{D} \boldsymbol{\epsilon}^{e}: \mathbf{E}^{e}: \dot{\boldsymbol{\epsilon}}
\end{array}\right] .
$$

The formulation of damage and plasticity, with different loading functions, is similar to multi-surface plasticity models and the consistency conditions may be combined to form the matrix format of the consistency conditions in the following manner:

$$
\left[\begin{array}{ll}
A_{11} & A_{12} \\
A_{21} & A_{22}
\end{array}\right]\left[\begin{array}{l}
\dot{\lambda} \\
\dot{D}
\end{array}\right]=\left[\begin{array}{l}
B_{1} \\
B_{2}
\end{array}\right]
$$

This results in a Linear Complementarity Problem (LCP) in the form:

$$
\dot{\mathbf{f}} \geq 0, \quad \dot{\lambda} \geq 0, \quad \dot{\mathbf{f}} \cdot \dot{\boldsymbol{\lambda}}=0 \Rightarrow \mathbf{A} \dot{\boldsymbol{\lambda}}-\mathbf{B} \geq 0, \quad \dot{\boldsymbol{\lambda}} \geq 0, \quad \dot{\lambda} \cdot(\mathbf{A} \dot{\boldsymbol{\lambda}}-\mathbf{B})=0
$$

and, in order to have a unique solution for $\dot{\boldsymbol{\lambda}}$, it suffices to show that $\mathbf{A}$ is a P-matrix, i.e. every principal minor of matrix $\mathbf{A}$ is greater than or equal to zero or $\mathbf{A}$ is positive definite.

These conditions may be expressed as:

$$
A_{11}>0, \quad A_{22}>0, \quad A_{11} A_{22}-A_{12} A_{21}>0
$$

and since in this special format $A_{12}$ is equal to zero, the third condition vanishes and the remaining conditions could be so explicated:

$$
\mathbf{n}: \mathbf{E}^{e}: \mathbf{m}-l\|\mathbf{m}\|>0 \text { and } \quad-\mathrm{r}_{0}(\mathrm{p}-1)(1-\mathrm{D})^{\mathrm{p}-2}>0 .
$$

The plastic multiplier and damage rate may be expressed in terms of matrices $\mathbf{A}$ and $\mathbf{B}$ as follows:

$$
\left[\begin{array}{l}
\dot{\lambda} \\
\dot{D}
\end{array}\right]=\frac{1}{A_{11} A_{22}-A_{12} A_{21}}\left[\begin{array}{cc}
A_{22} & -A_{12} \\
-A_{21} & A_{11}
\end{array}\right]\left[\begin{array}{l}
B_{1} \\
B_{2}
\end{array}\right] .
$$


Now that the background formulations for plasticity, damage and the coupling between these models is described, an implicit backward Euler method in conjunction with Newton-Raphson method is used to find the updated values of the unknowns.

\subsubsection{Elastoplastic-Damage Tangent Operator}

The elastoplastic-damage tangent operator can be achieved analytically based on the aforementioned equations and it has three formats based on the loading conditions; it could be elasto-damage, elastoplastic and elastoplasticdamage tangent operator. The elasto-damage and elastoplastic tangent operators are explicitly derived in the last sections; in this one the elastoplasticdamage one is derived for both Boltzmann and Cosserat continua.

Elastoplastic-Damage Tangent Operator for Boltzmann Continuum.

In presence of damage and plasticity the tangent operator can be derived from the following expression:

$$
\dot{\boldsymbol{\sigma}}=(1-D) \dot{\overline{\boldsymbol{\sigma}}}-\overline{\boldsymbol{\sigma}} \dot{D}
$$

where $\dot{\overline{\boldsymbol{\sigma}}}$ assumes the form here reported

$$
\dot{\overline{\boldsymbol{\sigma}}}=\mathbf{E}^{e p}: \dot{\boldsymbol{\epsilon}}
$$


Therefore it is possible to obtain the elastoplastic-damage continuum tangent operator as here reported:

$$
\begin{aligned}
& \mathbf{E}^{e p d}=(1-D) \mathbf{E}^{e p}-\overline{\boldsymbol{\sigma}} \otimes \mathbf{E}_{2^{n d}}^{e d}=(1-D)\left[\mathbf{E}^{e}-\frac{\mathbf{E}^{e}: \mathbf{m} \otimes \mathbf{n}: \mathbf{E}^{e}}{\mathbf{n}: \mathbf{E}^{e}: \mathbf{m}-l\|\mathbf{m}\|}\right]- \\
& \overline{\boldsymbol{\sigma}} \otimes\left[\frac{p_{D} \boldsymbol{\epsilon}^{e}:\left(\mathbf{E}^{e}-\frac{\mathbf{E}^{e}: \mathbf{m} \otimes \mathbf{n}: \mathbf{E}^{e}}{\mathbf{n}: \mathbf{E}^{e}: \mathbf{m}-l\|\mathbf{m}\|}\right)+c \frac{\overline{\boldsymbol{\sigma}}: \mathbf{m} \otimes \mathbf{n}: \mathbf{E}^{e}}{r_{0}(p-1)(1-D)^{p-2}}}{\mathbf{n}: \mathbf{E}^{e}: \mathbf{m}-l\|\mathbf{m}\|}\right]
\end{aligned}
$$

where $\mathbf{E}_{2^{\text {nd }}}^{e d}$ is a second order tensor.

\section{Elastoplastic-Damage Tangent Operator for Cosserat Continuum.}

As done for the classical continuum, but now considering that strains, stresses and the stiffness matrix are all matrices that contain both the components of classical and Cosserat continua, i.e.,

$$
\boldsymbol{\epsilon}^{e}=\left[\begin{array}{c}
\boldsymbol{\epsilon}^{e} \\
l \boldsymbol{\kappa}^{e}
\end{array}\right], \overline{\boldsymbol{\sigma}}=\left[\begin{array}{c}
\overline{\boldsymbol{\sigma}}^{u} \\
\overline{\boldsymbol{\mu}}
\end{array}\right], \mathbf{m}=\left[\begin{array}{l}
\mathbf{m}^{u} \\
\mathbf{m}^{\omega}
\end{array}\right], \mathbf{n}=\left[\begin{array}{l}
\mathbf{n}^{u} \\
\mathbf{n}^{\omega}
\end{array}\right], \mathbf{E}^{e p}=\left[\begin{array}{ll}
\mathbf{E}_{u u}^{e p} & \mathbf{E}_{u \omega}^{e p} \\
\mathbf{E}_{\omega u}^{e p} & \mathbf{E}_{\omega \omega}^{e p}
\end{array}\right]
$$

the elastoplasto-damage tangent continuum operator for Cosserat continuum is obtained:

$$
\mathbf{E}^{e p d}=(1-D)\left[\begin{array}{ll}
\mathbf{E}_{u u}^{e p} & \mathbf{E}_{u \omega}^{e p} \\
\mathbf{E}_{\omega u}^{e p} & \mathbf{E}_{\omega \omega}^{e p}
\end{array}\right]+\left[\begin{array}{ll}
\mathbf{E}_{u u}^{d} & \mathbf{E}_{u \omega}^{d} \\
\mathbf{E}_{\omega u}^{d} & \mathbf{E}_{\omega \omega}^{d}
\end{array}\right]
$$


where the components of the second tensor are so defined:

$$
\begin{aligned}
& \mathbf{E}_{u u}^{d}= \overline{\boldsymbol{\sigma}}^{u} \otimes\left[\frac{p_{D}}{R_{D}}\left(\boldsymbol{\epsilon}^{e}: \mathbf{E}_{u u}^{e p}+l \boldsymbol{\kappa}^{e}: \mathbf{E}_{\omega u}^{e p}\right)\right]+ \\
& \overline{\boldsymbol{\sigma}}^{u} \otimes[ {\left[\frac{c}{R_{D} H_{c}^{p}}\left(\overline{\boldsymbol{\sigma}}^{u}: \mathbf{m}^{u}+\frac{\overline{\boldsymbol{\mu}}}{l}: \mathbf{m}^{\omega}\right)\left(\mathbf{n}^{u}: \mathbf{E}_{u u}^{e}+\mathbf{n}^{\omega}: \mathbf{E}_{\omega u}^{e}\right)\right], } \\
& \mathbf{E}_{u \omega}^{d}= \overline{\boldsymbol{\sigma}}^{u} \otimes\left[\frac{p_{D}}{R_{D}}\left(\boldsymbol{\epsilon}^{e}: \mathbf{E}_{u \omega}^{e p}+l \boldsymbol{\kappa}^{e}: \mathbf{E}_{\omega \omega}^{e p}\right)\right]+ \\
& \overline{\boldsymbol{\sigma}}^{u} \otimes\left[\frac{c}{R_{D} H_{c}^{p}}\left(\overline{\boldsymbol{\sigma}}^{u}: \mathbf{m}^{u}+\frac{\overline{\boldsymbol{\mu}}}{l}: \mathbf{m}^{\omega}\right)\left(\mathbf{n}^{u}: \mathbf{E}_{u \omega}^{e}+\mathbf{n}^{\omega}: \mathbf{E}_{\omega \omega}^{e}\right)\right], \\
& \mathbf{E}_{\omega u}^{d}=\frac{\overline{\boldsymbol{\mu}}}{l} \otimes\left[\frac{p_{D}}{R_{D}}\left(\boldsymbol{\epsilon}^{e}: \mathbf{E}_{u u}^{e p}+l \boldsymbol{\kappa}^{e}: \mathbf{E}_{\omega u}^{e p}\right)\right]+ \\
& \overline{\boldsymbol{\sigma}}^{u} \otimes\left[\frac{c}{R_{D} H_{c}^{p}}\left(\overline{\boldsymbol{\sigma}}^{u}: \mathbf{m}^{u}+\frac{\overline{\boldsymbol{\mu}}}{l}: \mathbf{m}^{\omega}\right)\left(\mathbf{n}^{u}: \mathbf{E}_{u u}^{e}+\mathbf{n}^{\omega}: \mathbf{E}_{\omega u}^{e}\right)\right], \\
& \mathbf{E}_{\omega \omega}^{d}=\frac{\overline{\boldsymbol{\mu}}}{l} \otimes\left[\frac{p_{D}}{R_{D}}\left(\boldsymbol{\epsilon}^{e}: \mathbf{E}_{u \omega}^{e p}+l \boldsymbol{\kappa}^{e}: \mathbf{E}_{\omega \omega}^{e p}\right)\right]+ \\
& \overline{\boldsymbol{\sigma}}^{u} \otimes\left[\frac{c}{R_{D} H_{c}^{p}}\left(\overline{\boldsymbol{\sigma}}^{u}: \mathbf{m}^{u}+\frac{\overline{\boldsymbol{\mu}}}{l}: \mathbf{m}^{\omega}\right)\left(\mathbf{n}^{u}: \mathbf{E}_{u \omega}^{e}+\mathbf{n}^{\omega}: \mathbf{E}_{\omega \omega}^{e}\right)\right] .
\end{aligned}
$$

It should be noted that $R_{D}$ is defined as

$$
R_{D}=-r_{0}(p-1)(1-D)^{p-2} .
$$

\section{Basic Concepts of Localization Analysis}

As mentioned in the introduction, localization analysis is based on bifurcation studies of the acoustic tensor of the constitutive relationship describing the mechanical concrete properties in terms of tangential (rate-type) stressstrain relationships.

In this section the fundamental arguments behind localization analysis of a work hardening-softening solid will be reviewed and its propensity to create 
weak and strong discontinuities in form of discrete fractures will be interrogated.

It can be noted that the tangent stiffness operator might experience singularities depending on the degree of non-associativity and the degree of hardening/softening. Under full strain control the tangential stiffness will lead to single-valued predictions, however this is not the case when the constitutive relationship is inverted or partially inverted. The singularity of the constitutive operator will lead to loss of uniqueness when $\operatorname{det}\left(\mathbf{E}^{e p d}\right)=0$. In addition to the question of loss of uniqueness, which typically arises in softening formulations, there is another critical material condition which is referred to as loss of ellipticity (or loss of hyperbolicity, which is the Hadamard condition in the context of wave propagation). This condition probes whether the constitutive operator leads to non-propagating standing wave situations when the acoustic tensor exhibits a singularity. The Hadamard condition [13] leads to localization analysis, which is the terminology introduced by Rudnicky and Rice [11] in their seminal paper.

In this case we are looking for the formation of weak discontinuities in the field of strain rates, hence we are searching for possible jump conditions of the velocity gradients across a singularity surface with the properties,

$$
\begin{aligned}
& \dot{\mathbf{u}}^{+}=\dot{\mathbf{u}}^{-} \Rightarrow[[\dot{\mathbf{u}}]]=0, \\
& \nabla \dot{\mathbf{u}}^{+} \neq \nabla \dot{\mathbf{u}}^{-} \Rightarrow[[\nabla \dot{\mathbf{u}}]] \neq 0
\end{aligned}
$$

where the '+' and '-' signs refer to the positive and negative sides of the discontinuity surface.

In other terms, the velocity gradient is a rank-one tensor that can be represented by the cross product of two vectors; the normal vector $\mathbf{N}$ to the 
discontinuity surface and the polarization vector $\mathbf{M}$ of particle movement. In fracture mechanics, this refers to mode I fracture in the case of crack opening, when $\mathbf{M}$ is collinear with $\mathbf{N}$, and to mode II fracture for sliding when $\mathbf{M}$ is perpendicular to $\mathbf{N}$.

The corresponding jump of the strain rate satisfies the Maxwell condition of compatibility across a discontinuity surface, when the velocity gradient exhibits a jump of the form,

$$
[[\nabla \dot{\mathbf{u}}]]=\dot{\gamma} \cdot \mathbf{M} \otimes \mathbf{N}
$$

Equilibrium is satisfied by the Cauchy condition of traction continuity,

$$
[[\dot{\mathbf{t}}]]=\dot{\mathbf{t}}^{+}-\dot{\mathbf{t}}^{-}=0
$$

which leads to the following bifurcation condition:

$$
\begin{aligned}
& {[[\dot{\mathbf{t}}]]=\mathbf{N} \cdot[[\dot{\boldsymbol{\sigma}}]]=\mathbf{N} \cdot\left[\left[\mathbf{E}^{*}: \dot{\boldsymbol{\epsilon}}\right]\right]=\mathbf{N} \cdot \mathbf{E}^{*}:[[\dot{\boldsymbol{\epsilon}}]] } \\
\Rightarrow & {[[\dot{\mathbf{t}}]]=\dot{\gamma} \cdot \mathbf{N} \cdot \mathbf{E}^{*} \cdot \mathbf{N} \cdot \mathbf{M}=\dot{\gamma} \cdot \mathbf{Q}^{*} \cdot \mathbf{M}=0 }
\end{aligned}
$$

where $\mathrm{Q}^{*}$ could be the elastoplastic, elastodamage or elastoplastic damage localization tensor and is defined as:

$$
\mathbf{Q}^{*}=\mathbf{N} \cdot \mathbf{E}^{*} \cdot \mathbf{N}
$$

The underlying eigenvalue problem requires the search for possible singularities of the tangential localization tensor, i.e.,

$$
\operatorname{det}\left(\mathbf{Q}^{*}\right)=0 \Rightarrow \lambda_{\min }\left(\mathbf{Q}^{*}\right)=0
$$

Based on equation (62), the loss of ellipticity occurs when the minimum eigenvalue of the acoustic tensor is equal to zero. 
In the case of elasto-plasticity the localization tensor is comprised of an elastic and a plastic contribution and may be expressed as:

$$
\mathbf{Q}^{e p}=\mathbf{Q}^{e}-\frac{\mathbf{N} \cdot \mathbf{E}^{e}: \mathbf{m} \otimes \mathbf{n}: \mathbf{E}^{e} \cdot \mathbf{N}}{H^{p}}
$$

where the special format of the second term on the rhs represents the plastic contribution that may be transformed into a scalar format for localization. Equation (63) can be reformulated as:

$$
\mathbf{Q}^{e p}=\mathbf{Q}^{e}-\frac{\mathbf{e}_{m} \otimes \mathbf{e}_{n}}{H^{p}}, \quad \mathbf{e}_{m}=\mathbf{N} \cdot \mathbf{E}^{e}: \mathbf{m}, \quad \mathbf{e}_{n}=\mathbf{n}: \mathbf{E}^{e} \cdot \mathbf{N} .
$$

Based on Ottosen and Runesson [32] the generalized eigenvalue problem is defined as,

$$
\operatorname{det}\left[\left(\mathbf{Q}^{e}\right)^{-1} \cdot \mathbf{Q}^{e p}\right]=\operatorname{det}\left[\mathbf{1}-\left(\mathbf{Q}^{e}\right)^{-1} \cdot \frac{\mathbf{e}_{m} \otimes \mathbf{e}_{n}}{H^{p}}\right]
$$

therefore the solution of the lowest eigenvalue of equation (65) becomes:

$$
\lambda_{\min }\left[\left(\mathbf{Q}^{e}\right)^{-1} \cdot \mathbf{Q}^{e p}\right]=\mathbf{1}-\frac{\mathbf{e}_{m} \cdot\left(\mathbf{Q}^{e}\right)^{-1} \cdot \mathbf{e}_{n}}{H^{p}+\mathbf{n}: \mathbf{E}^{e}: \mathbf{m}}
$$

and the critical hardening modulus indicating loss of ellipticity may be expressed as:

$$
H_{p}^{c r i t}=\mathbf{e}_{n} \cdot\left(\mathbf{Q}^{e}\right)^{-1} \cdot \mathbf{e}_{m}-\mathbf{n}: \mathbf{E}^{e}: \mathbf{m} .
$$

The same procedure can be followed for the elasto-damage material behavior; in this case the elasto-damage localization tensor becomes:

$$
\mathbf{Q}^{e d}=(1-D) \mathbf{Q}^{e}-p_{D} \frac{\mathbf{N} \cdot \mathbf{E}^{e}: \boldsymbol{\epsilon}^{e} \otimes \boldsymbol{\epsilon}^{e}: \mathbf{E}^{e} \cdot \mathbf{N}}{r_{0}(p-1)(1-D)^{p-2}}
$$

where it can be reformulated as,

$$
\mathbf{Q}^{e d}=(1-D) \mathbf{Q}^{e}-p_{D} \frac{\mathbf{e}_{1}^{d} \otimes \mathbf{e}_{2}^{d}}{r_{0}(p-1)(1-D)^{p-2}}
$$


and where $\mathbf{e}_{1}^{d}$ and $\mathbf{e}_{2}^{d}$ are expressed as,

$$
\mathbf{e}_{1}^{d}=\mathbf{N} \cdot \mathbf{E}^{e}: \boldsymbol{\epsilon}^{e} \quad \text { and } \quad \mathbf{e}_{2}^{d}=\boldsymbol{\epsilon}^{e}: \mathbf{E}^{e} \cdot \mathbf{N}
$$

In this case the eigenvalue problem is so defined:

$$
\operatorname{det}\left[\left(\mathbf{Q}^{e}\right)^{-1} \cdot \mathbf{Q}^{e d}\right]=(1-D) \operatorname{det}\left[\mathbf{1}-p_{D}\left(\mathbf{Q}^{e}\right)^{-1} \cdot \frac{\mathbf{e}_{1}^{d} \otimes \mathbf{e}_{2}^{d}}{r_{0}(p-1)(1-D)^{p-2}}\right]
$$

and the lowest eigenvalue of equation (71) becomes:

$$
\lambda_{\min }\left(\left(\mathbf{Q}^{e}\right)^{-1} \cdot \mathbf{Q}^{e d}\right)=(1-D) \mathbf{1}-p_{D} \frac{\mathbf{e}_{1}^{d} \cdot\left(\mathbf{Q}^{e}\right)^{-1} \cdot \mathbf{e}_{2}^{d}}{H^{d}}
$$

obtaining a critical hardening modulus, which indicates loss of ellipticity, in the form,

$$
H_{c r i t}^{d}=\frac{p_{D}}{(1-D)} \mathbf{e}_{1}^{d} \cdot\left(\mathbf{Q}^{e}\right)^{-1} \cdot \mathbf{e}_{2}^{d} .
$$

For the elastoplasto-damage case, since the analytical solution is very com-

plicated, numerical analysis is used to find the critical eigenvalues of the localization tensor.

\subsection{Localization Analysis for Cosserat Continuum}

Weak discontinuities in a Cosserat continuum can not only appear in the strain rates, but they could be also present for the rate of micro-curvatures. Consequently the displacements (and displacement rates) and rotations (and rate of rotations) are continuous, while the gradient of the displacement and rotation fields are discontinuous. This is expressed as:

$$
\begin{aligned}
& \nabla \dot{\mathbf{u}}^{+} \neq \nabla \dot{\mathbf{u}}^{-} \Rightarrow[[\nabla \dot{\mathbf{u}}]]=\nabla \dot{\mathbf{u}}^{+}-\nabla \dot{\mathbf{u}}^{-} \neq 0, \\
& \nabla \dot{\boldsymbol{\omega}}^{+} \neq \nabla \dot{\boldsymbol{\omega}}^{-} \Rightarrow[[\nabla \dot{\boldsymbol{\omega}}]]=\nabla \dot{\boldsymbol{\omega}}^{+}-\nabla \dot{\boldsymbol{\omega}}^{-} \neq 0 .
\end{aligned}
$$


Based on equations (74a) and (74b) and Maxwell compatibility condition, the jump in the rate of strain and micro-curvature fields becomes,

$$
[[\dot{\boldsymbol{\epsilon}}]]=\dot{\gamma}^{u} \cdot \mathbf{N} \otimes \mathbf{M}^{u} \quad \text { and } \quad[[\dot{\boldsymbol{\epsilon}}]]=\dot{\gamma}^{\omega} \cdot \mathbf{N} \otimes \mathbf{M}^{\omega}
$$

where $\mathbf{M}^{u}$ and $\mathbf{M}^{\omega}$ are the polarization vectors for strain and micro-curvature rates and $\dot{\gamma}^{u}$ and $\gamma^{\omega}$ are the undetermined magnitudes of jumps.

According to Cauchy Lemma the traction rate vectors of stresses and couple stresses should remain continuous on the discontinuity surface, which would result in the first localization condition,

$$
\operatorname{det}(\mathbf{Q})^{*}=\left[\begin{array}{ll}
\mathbf{Q}_{u u}^{*} & \mathbf{Q}_{u \omega}^{*} \\
\mathbf{Q}_{\omega u}^{*} & \mathbf{Q}_{\omega \omega}^{*}
\end{array}\right]=0 .
$$

According to Willam et al. [27], the second localization condition rises from the balance of linear and angular momentum on the both sides of singularity surface and is so defined:

$$
\mathbf{e}:[[\dot{\boldsymbol{\sigma}}]]=\dot{S}^{u}+\dot{S}^{\omega}=0
$$

where the two scalar terms have the forms,

$$
\dot{S}^{u}=\dot{\gamma}^{u} \mathbf{e}:\left[\mathbf{E}_{u u}^{e p}:\left(\mathbf{N} \otimes \mathbf{M}^{u}\right)\right] \quad \text { and } \quad \dot{S}^{\omega}=\dot{\gamma}^{\omega} \mathbf{e}:\left[\mathbf{E}_{u \omega}^{e p}:\left(\mathbf{N} \otimes \mathbf{M}^{\omega}\right)\right]
$$

The first and second localization conditions have to be satisfied simultaneously in order to admit loss of ellipticity.

In this work numerical localization analysis for Cosserat continuum is performed and the results are presented in the following sections. 


\section{Experimental Investigations}

Concrete cylinders of size $101.6 \times 101.6 \times 203.2 \mathrm{~mm}$ are tested in compression under monotonic and cyclic loading. The mixture adopted for the specimens is tabulated in Table 1; the values reported below are normalized with respect to the mass of cement content.

Table 1: Mixture of the concrete specimens.

\begin{tabular}{|l|c|c|c|c|}
\hline & Cement & Water & Sand & Aggregate \\
\hline Normalized Mass & 1 & 0.5 & 1.53 & 2.37 \\
\hline
\end{tabular}

In order to test 25 concrete samples, a Tinius Olsen compression-tension machine in displacement control has been adopted and a digital image correlation system has taken pictures of the specimens at a constant pace and has recorded the data on a computer.

The fractured specimen under cyclic loading is depicted in Figure 4, which shows axial splitting of failed specimen after reaching the maximum load resistance.

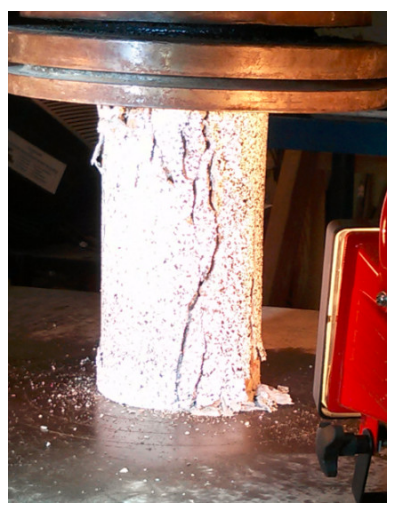

Figure 4: Failed concrete specimen. 
The corresponding stress-strain curves of the uniaxial and cyclic compression tests are presented in Figure 5.
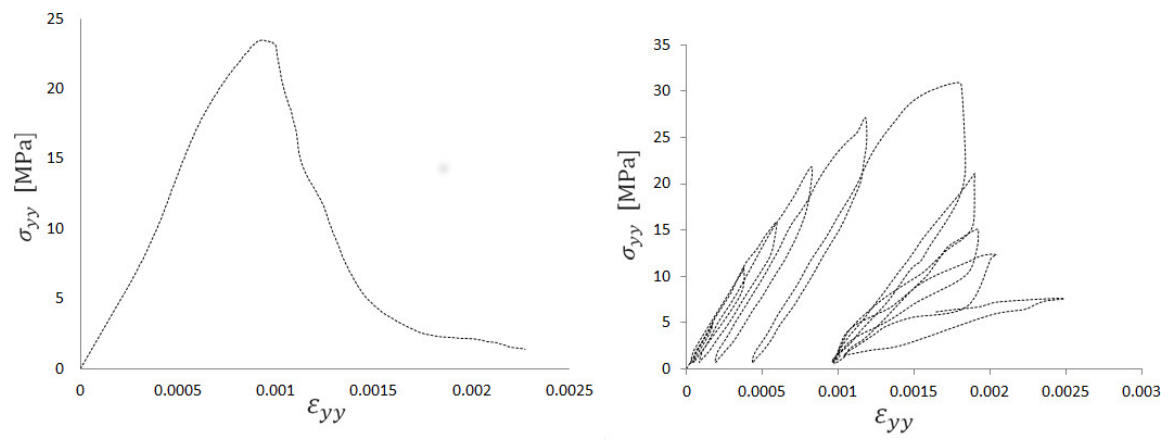

Figure 5: Stress strain curves: monotonic compression on the left; cyclic compression on the right.

\subsection{Digital Image Correlation Analysis}

The DIC setup used for this study, is a non-contact optical 3D metrology system in which the ARAMIS software analyzes, calculates and documents deformations at prescribed load steps [30].

There are several steps to accomplish in a typical measuring procedure using the DIC system, such as the preparation of the specimen and the experiment; the images recording and the processing of the recorded images using ARAMIS.

As first the surfaces of the sample facing the cameras, which must meet certain requirements like being smooth, are covered by a white paint and, subsequently, black random dots are sprayed to generate a speckle pattern. Once the sample is carefully painted and it has been inserted in the test 
setup, a pair of 12 magapixel Gigabit Ethernet Cameras with 50mm fixed local lenses is placed in front of the sample. The two cameras take pictures at presetted time intervals during the test and measure the movement of target points on the surface of the specimen. The first image corresponds to the undeformed or reference state.

After recording the digital images of the sample during the test, ARAMIS computes the motion of each point comparing the images at different states. To do this the software specifies on the initial image several square boxes known as facets or subsets, on the surface of the specimen, where each of these facets contains nn pixels (they are tipically in the range 5-20 pixels squares) of the target points and they are placed m pixels away from one another. The spatial position of the centre point of these subsets is calculated by averaging the positions of the corners. This post processing procedure of results allows to determine the strain and displacement fields.

The transverse displacement evolution of the concrete sample under compression for different stages of the experiment is depicted in Figure 6. In the right figures it can be observed that there is a jump, which confirms the cracking of the specimen at that stage. Indeed DIC displacement field observations result important to display axial crack formation in the postpeak response regime besides axial strain measurements at several segments of the perimeter with the help of virtual extensional strain gauges to extract nominal deformation values for specimen compaction. 

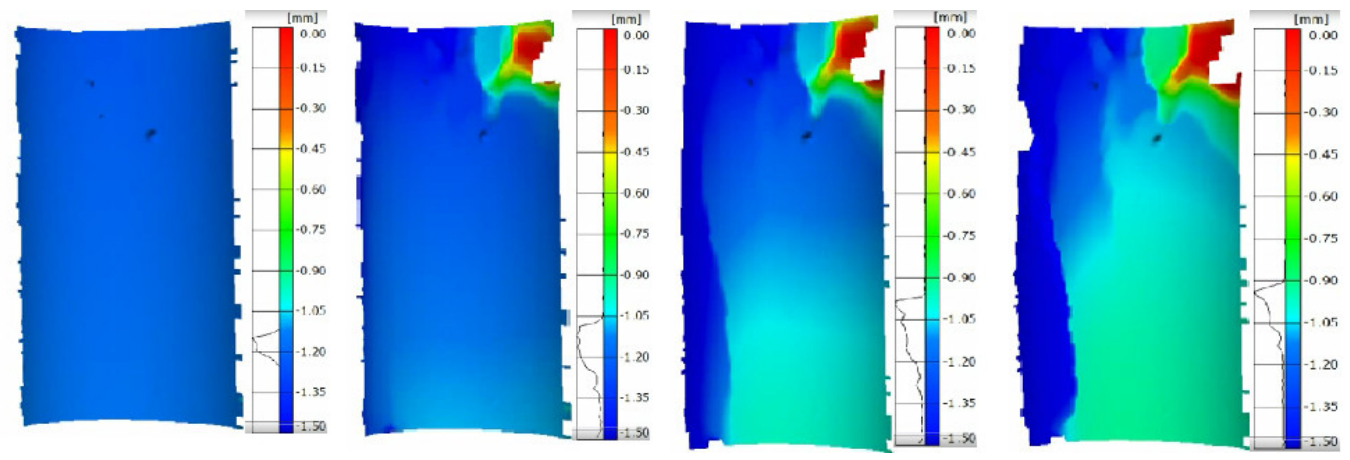

Figure 6: Evolution of the transverse displacement [mm] for different stages of the experiment.

The results of digital image correlation in Figure 7 illustrate the formation of lateral (circumferential strains) on the cylinder surface in support of the axial splitting mode of failure.
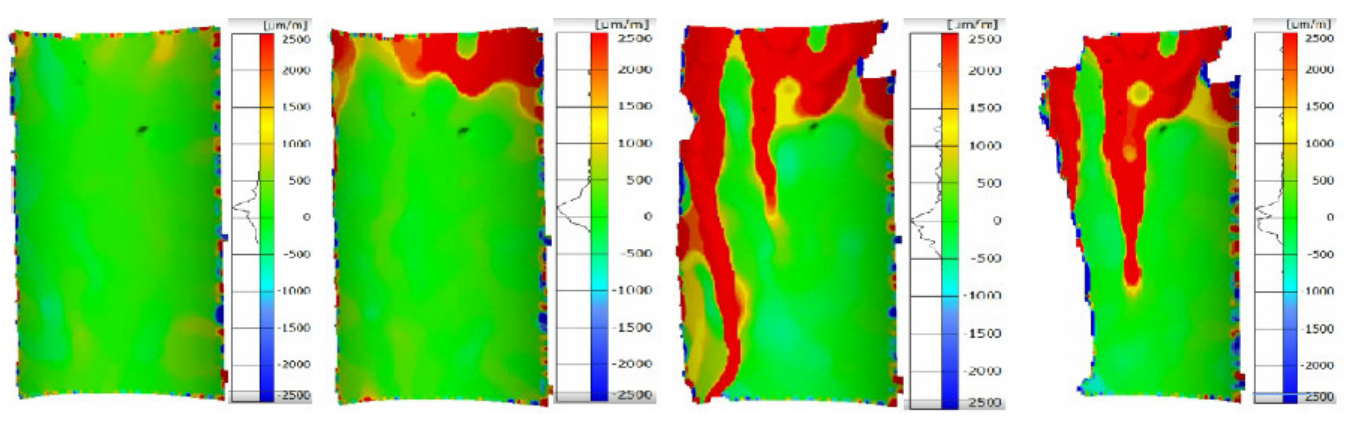

Figure 7: Evolution of the transverse strain for different stages of the experiment.

\section{Numerical Simulations}

In order to simulate concrete's behavior the experimental data (obtained by compression tests under monotonic and cyclic loading) are extracted and used in order to calibrate the numerical model, previously introduced in this 
work. The benefit of using plasticity models for concrete is that they are capable of simulating permanent deformations in the case of unloading, while the damage model will have an unloading path that returns to initial values of strains.

The performance of the model is evaluated for different load scenarios such as uniaxial compression, tension and simple shear. Subsequently the numerical localization analysis is performed for the damage, plasticity and damageplasticity models, where the differences of the localization properties of these models are discussed and assessed, based on the observations achieved by the experiments. Both Boltzmann and Cosserat continua are investigated to further study the localization properties.

It should be noted that the proposed model is coded in Matlab software [33]. The problems are solved using a nonlinear displacement control scheme. At each displacement step, for each element and Gauss point, the strains are calculated and a constitutive driver is called to calculate the updated values of stresses, plastic strains, damage variables and internal parameters. Afterwards the elastoplastic, elasto-damage or elastoplastic-damage tangent operators are calculated; the global stiffness matrix and force vectors are assembled for all the elements and finally the error associated with the displacement guess is calculated. If this error is less than the tolerance, it proceeds to the next step, otherwise the Newthon-Raphson method is used to update the displacement values. This procedure is repeated until the convergence is achieved. For the constitutive driver, a fully implicit backward Euler method along with the Newthon-Raphson method are utilized to solve for the unknowns; this procedure is adopted for both classical and Cosserat 
continua. The numerical algorithm is illustrated by a flow-chart in figure 8 .

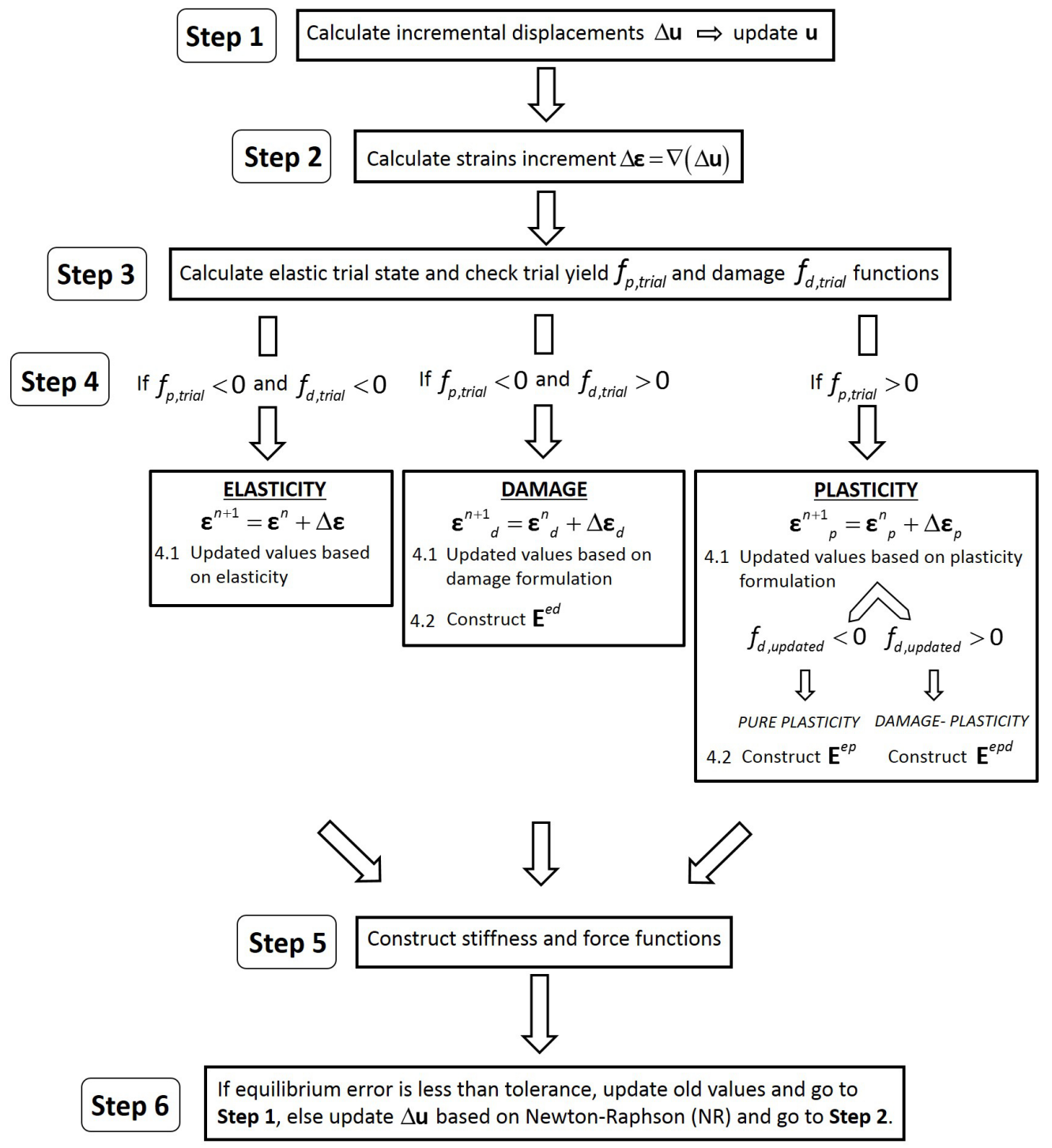

Note: damage and plasticity formulations solved for internal variables based on NR method

Figure 8: Numerical algorithm. 


\subsection{Model Calibration and Performance}

The damage-plasticity models are calibrated with the experimental results obtained from in-house experiments and the results are tabulated in Table 2 (the parameters $\epsilon_{e q, t}^{p}$ and $q_{h 0}$ appear in the hardening parameter $q_{h}$, while $t$ in the softening one $q_{s}$; see [7]). The main models parameters such as $E$, $\nu, f_{c}, f_{t}$ and the eccentricity $e$ were evaluated directly, while the secondary model parameters were adjusted by trial and error to obtain a reasonable fit on the axial response in cyclic compression.

Table 2: Mixture of the concrete specimens.

\begin{tabular}{c|c|c|c|c|c|c|c}
$E[M P a]$ & $\nu$ & $e$ & $\epsilon_{e q, t}^{p}$ & $q_{h 0}$ & $f_{c}[\mathrm{MPa}]$ & $f_{t}[\mathrm{MPa}]$ & $t$ \\
\hline 28000 & 0.15 & 0.52 & 0.00125 & 0.2 & 25 & 2.5 & 0.0025 \\
& $r_{0}$ & $p$ & $c_{d}$ & $P_{d}$ & $A$ & $B$ & \\
\cline { 2 - 7 } & 0.00018 & 0.65 & $1(0.01)$ & $1(0)$ & 0.5 & -12 &
\end{tabular}

The comparison of the stress-strain curve for cyclic compression test based on the calibrated data is depicted in Figure 9. It is observed that both classical and Cosserat damage-plasticity models are predicting the behavior of concrete. 


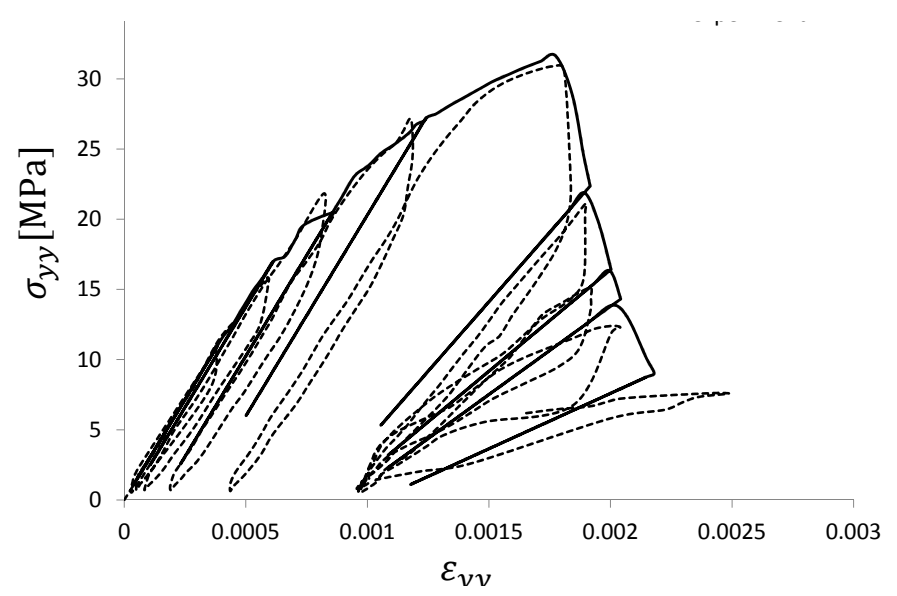

Figure 9: Calibrated numerical model for cyclic behavior.

Figure 9 illustrates the effect of damage which leads to negligible reduction of the initial stiffness property in the pre-peak regime, but which becomes very noticeable in the post-peak regime especially close to residual load resistance. All of the subsequent localization studies were performed at peak resistance of specific load histories under mixed control to activate the effect of damage on the localization properties as compared to the plastic contribution.

The performance of this damage-plasticity formulation in the case of uniaxial compression, tension and simple shear is depicted in figure 10. 

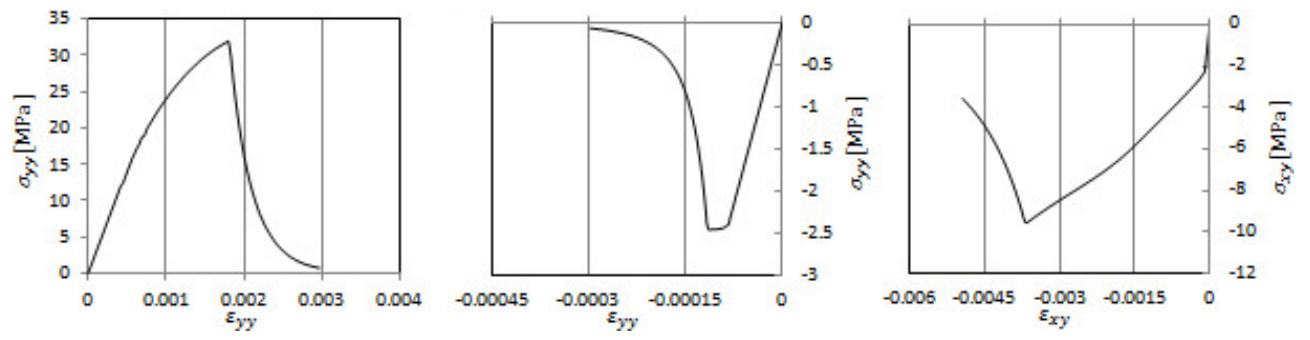

Figure 10: Stress-strain behavior: a) uniaxial compression; b) uniaxial tension; c) simple shear.

\subsection{Numerical Localization Analysis}

The numerical localization analysis is implemented inside the displacement loop. For every step the normalized value of the determinant of the localization tensor is recorded and finally the localization graphs of the damage, plasticity and damage-plasticity models are plotted. It should be noted that the localization analysis is performed assuming that all the components of the tangent operator are present. In the case of Cosserat continuum it is assumed that only the curvatures and micro-rotations of the loading plane are present.

The figures will report the localization results at the first step of plasticity, damage or damage plasticity repsectively. This means that, for example, in the damage-plasticity case, once the material becomes plastic, and then as soon as damage is also activated, the data are recorded and the localization tensor has been determined.

\subsection{First Localization Condition Investigations}




\subsubsection{Uniaxial Tension}

Figure 11 illustrates the variation, in the case of uniaxial tension, of the normalized localization tensor for different values of alpha that measure the orientation of the normal vector $\mathbf{N}$ with regard to the direction of minor principal stress.

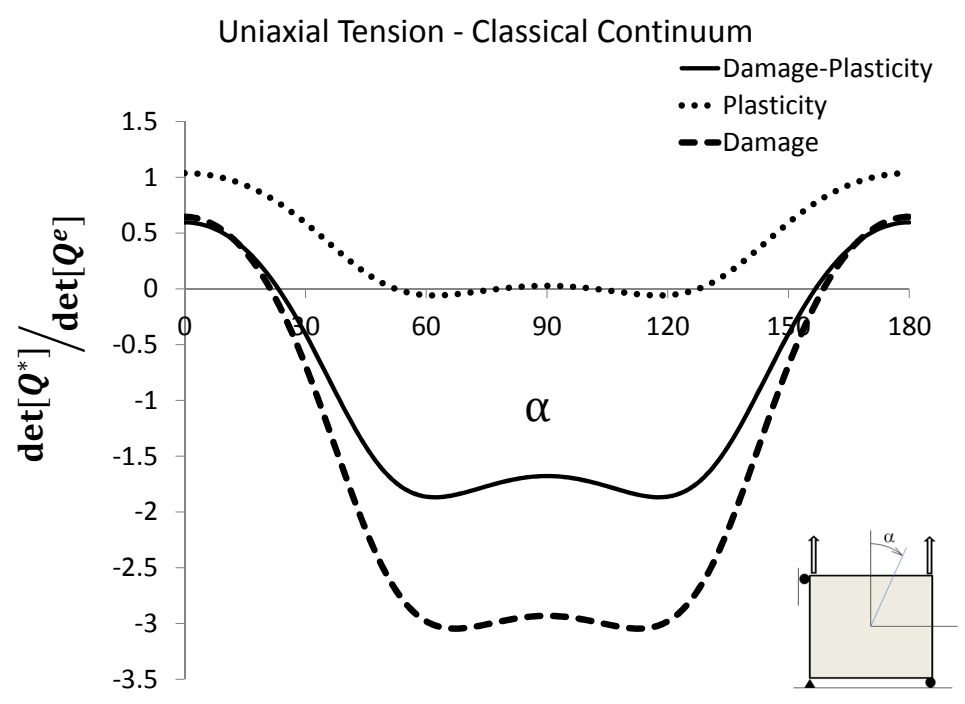

Figure 11: Localization analysis of Willam-Warnke and damage formulation under uniaxial tension.

By the result, it is possible to notice that the addition of the scalar damage model leads to much more pronounced degradation of the localization tensor when compared to the elasto-plastic contribution; in fact for the calibrated parameters, the normalized value of the determinant is mostly negative in the case of damage and damage-plasticity. The critical angle associated with this model is $\alpha=62^{\circ}$ for damage-plasticity and is $\alpha=67^{\circ}$ for damage in the $n_{1}-n_{2}$ plane. The plasticity model in tension has more stable localization 
properties and the minimum eigenvalue associated with it is $\alpha=62^{\circ}$. In the case of the Cosserat continua and uniaxial tension, the localization graphs are depicted in figure 12. It is observed that still the criticality of the damage

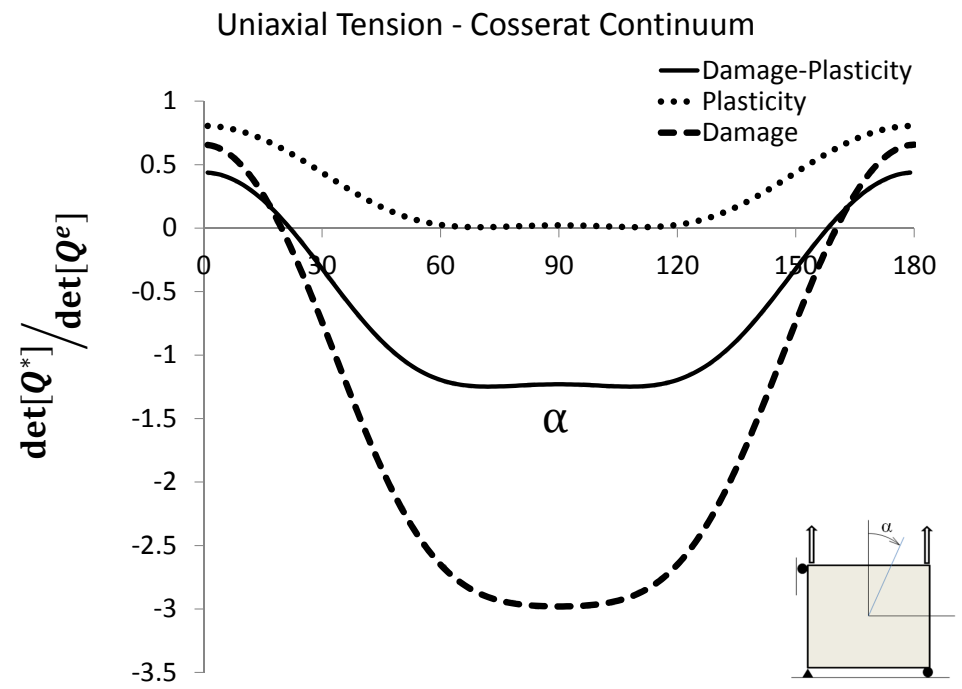

Figure 12: Localization analysis of Willam-Warnke and damage formulation under uniaxial tension for Cosserat continuum.

formulation is more in comparison to plasticity. It is observed that for the Cosserat shear modulus $G_{c}$ being half the shear modulus of a classical continuum $G$, the critical angle associated with plasticity is $\alpha=66^{\circ}$, the critical angle for damage-plasticity is $\alpha=63^{\circ}$ and the critical angle for damage is $\alpha=67^{\circ}$.

In order to study the effect of the tensile strength on the localization properties of the classical continuum, $e$ is set to 1 , and $f_{t}$ is changed. This is studied for $f_{t}$ being two times greater than the compressive strength to it being ten percent of the compressive strength. The results of this study are depicted in figure 13. It is observed that by increasing the ratio between the tensile and 


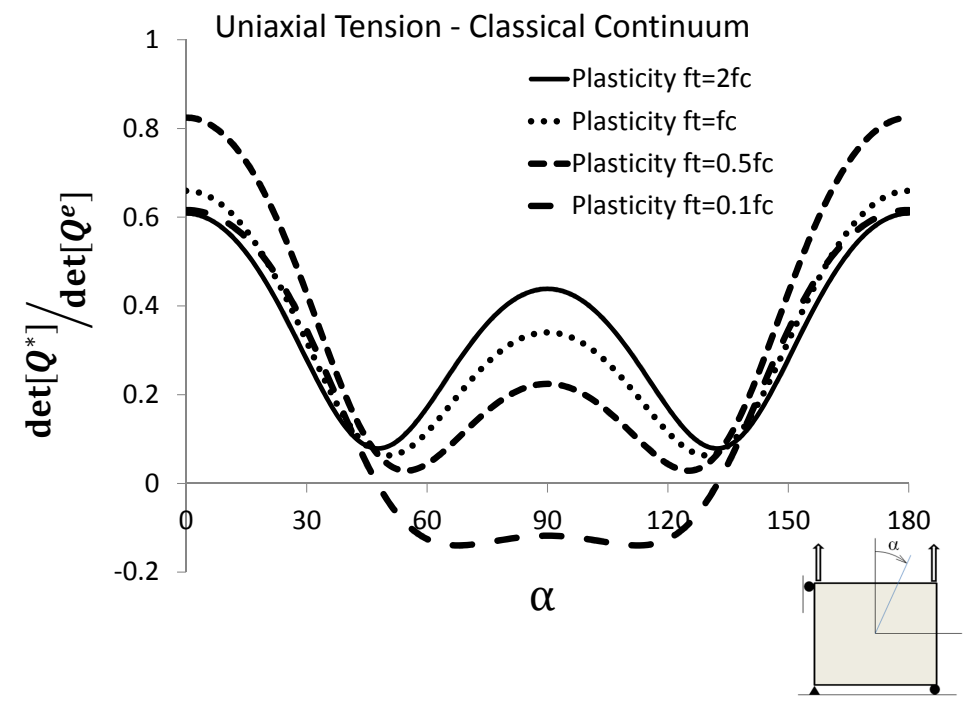

Figure 13: Localization analysis of Willam-Warnke plasticity under uniaxial tension for different values of $f_{t}$.

compressive strengths, the critical localization angle is shifted and the results become closer to the ones of the von Mises plasticity. The angles associated with these analyses are $\alpha=68^{\circ}, 55^{\circ}, 50^{\circ}, 48^{\circ}$ for $f_{t}$ being $0.1 f_{c}, 0.5 f_{c}, f_{c}$ and $f_{t}=2 f_{c}$ respectively. It should be also noted that the higher ratio reduces the susceptibility of the material to localized failure and loss of ellipticity.

\subsubsection{Uniaxial Compression}

In the compression test, since the damage model used here is not activated in the absence of plasticity, there is no pure damage case in compression test. Therefore localization analysis is performed only for plasticity and damage-plasticity. The critical angles associated with plasticity and damageplasticity are $\alpha=39^{\circ}$ and $\alpha=43^{\circ}$ correspondingly. The localization results for damage-plasticity and plasticity are depicted in figure 14 . 
The same analysis is performed for Cosserat continua and as mentioned before the pure damage case is not present for compression. The corresponding critical angles associated with plasticity and damage plasticity for this model are $\alpha=33^{\circ}$ and $\alpha=40^{\circ}$ respectively. The localization results are depicted in figure 15.

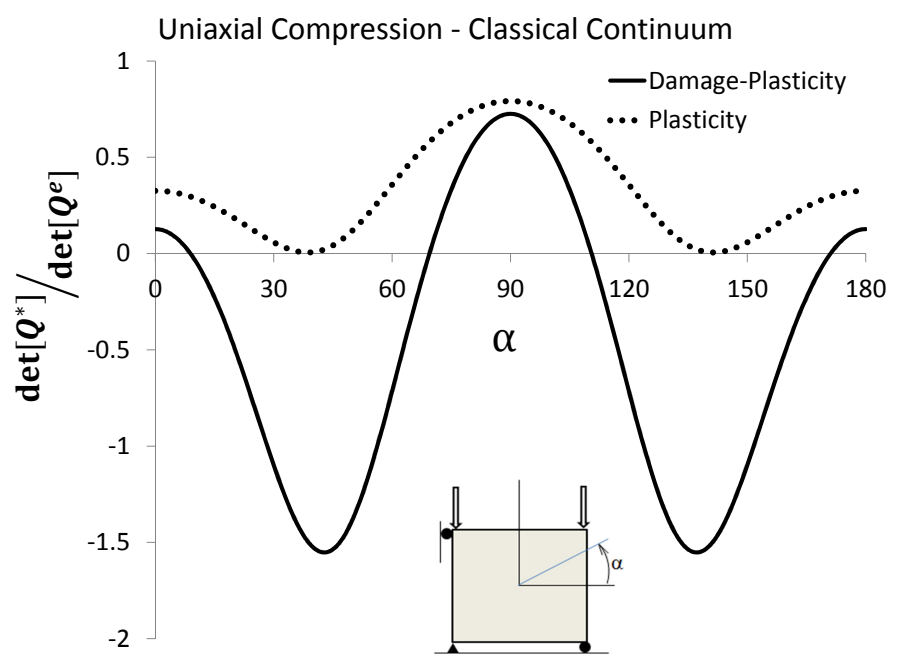

Figure 14: Localization analysis of Willam-Warnke and damage formulation under uniaxial compression. 


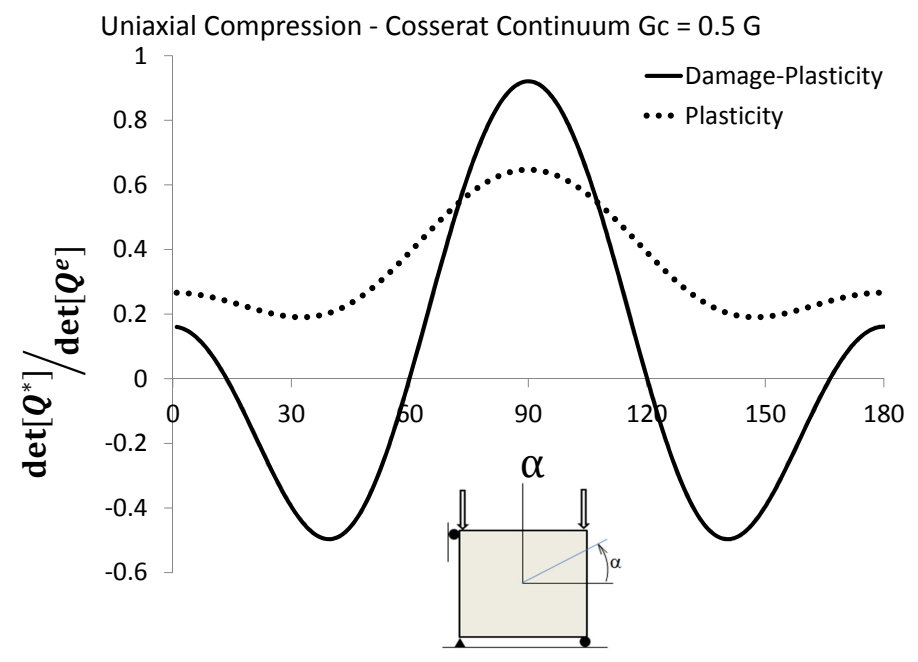

Figure 15: Localization analysis of Willam-Warnke and damage formulation under uniaxial compression for Cosserat continuum.

The localization properties of the damage-plasticity formulation for the case that the model is reduced to von Mises plasticity formulation are presented in Figure 16. A non-associative flow rule has been considered. It is observed that the critical angles are very close to the prediction of the von Mises plasticity formulation, i.e. 45 degrees. 


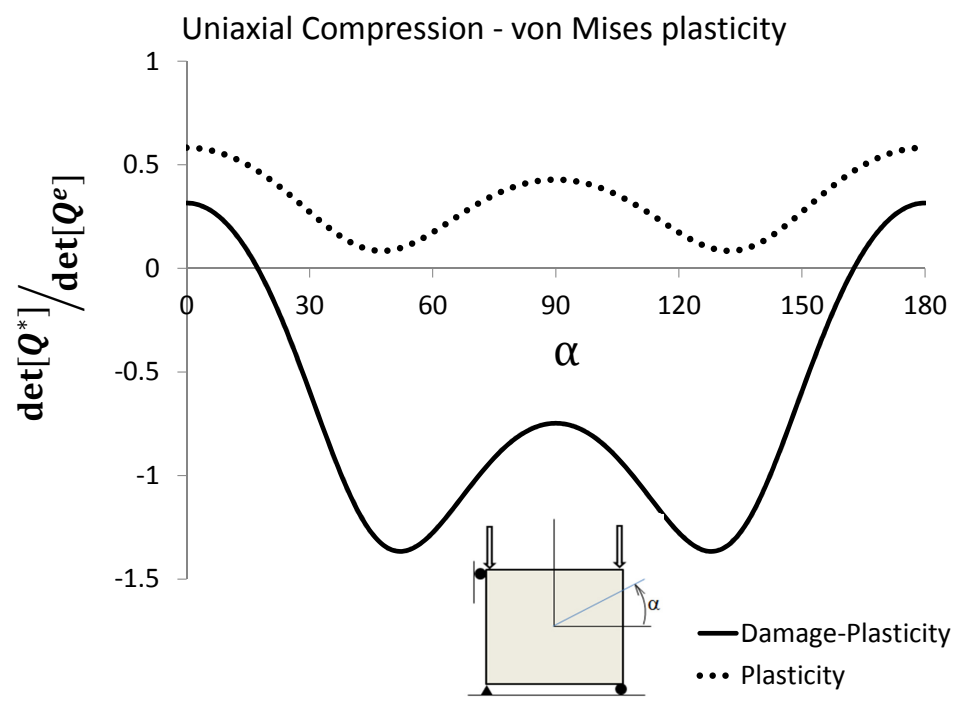

Figure 16: Localization analysis of Willam-Warnke and damage formulation under uniaxial compression for von Mises plasticity, $f_{c}=f_{t}$.

The effect of Cosserat shear modulus $\left(G_{c}\right)$ on the localization directions of the plasticity formulation has been investigated in Figure 17. It can be observed that for $G_{c}$ equal to zero the behavior is similar to the classical continuum, however as the value is closer to the shear modulus $(G)$ there is a significant change in the localization directions. For instance, when the Cosserat shear modulus is equal to $G$, the minimum normalized localization value would happen at 0 and 180 degrees. 


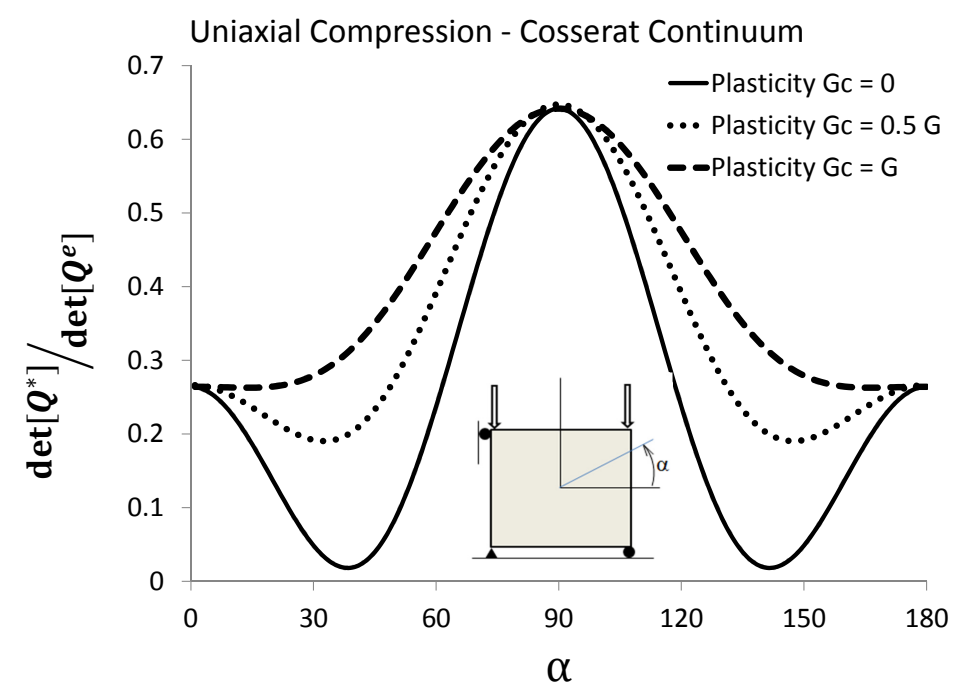

Figure 17: The effect of Cosserat shear modulus on the localization properties of plasticity formulation under uniaxial compression.

\subsubsection{Simple Shear, Pure Shear}

The loss of ellipticity for damage, plasticity and damage-plasticity formulation is studied for both simple and pure shear cases in the classical and Cosserat continua. The localization analyses for classical and Cosserat continua for the case of simple shear are depicted in figure 18. In the case of pure shear, the localization results are depicted in figure 19. The localization analysis of damage formulation displays the same trends for both classical and Cosserat continua. In the case of plasticity, it is observed that the behavior of the Cosserat continuum is no longer symmetrical which is due to the presence of couple stresses and the fact that $\sigma_{x y}$ is not equal to $\sigma_{y x}$. It is also observed that the damage-plasticity graphs in the case of Cosserat continuum are not symmetrical due to the presence of plasticity.

It should be noted that the angle $\alpha$ is assumed to start at 45 degrees for 
the cases of shear loading.

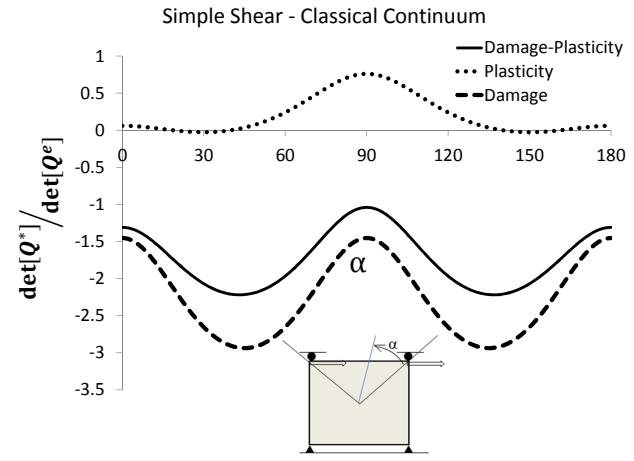

(a) Classical

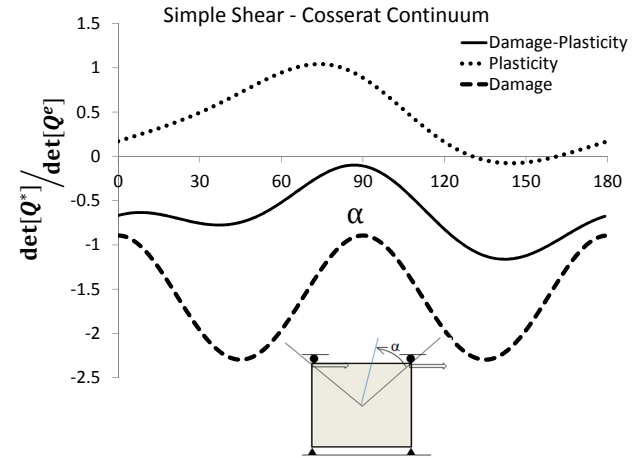

(b) Cosserat

Figure 18: Localization results of Willam-Warnke plasticity and damage for simple shear.

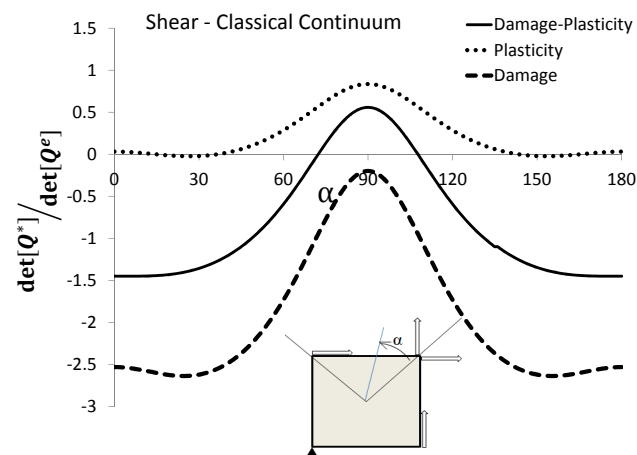

(a) Classical

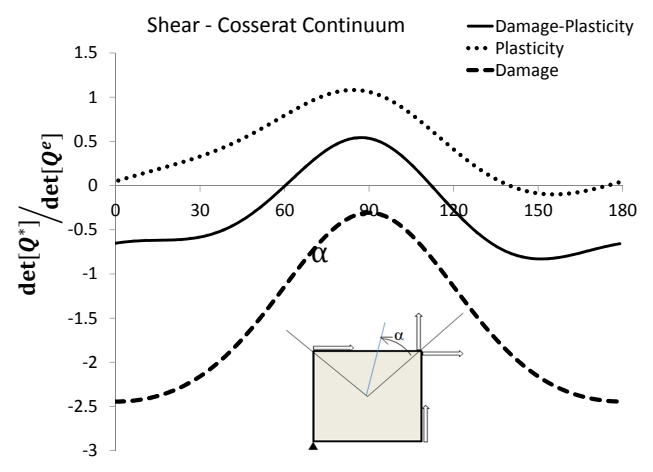

(b) Cosserat

Figure 19: Localization results of Willam-Warnke plasticity and damage for pure shear.

\subsubsection{Bi-Axial Tension}

In this section the effect of Cosserat continuum on the regularization of discontinuities in the Willam-Warnke plasticity and damage for bi-axial cases is studied. The comparison of the plasticity localization graphs for classical continuum and Cosserat continuum with $G_{c}$ being equal to $0.5 G$ and $G$ is depicted in figure 20. 


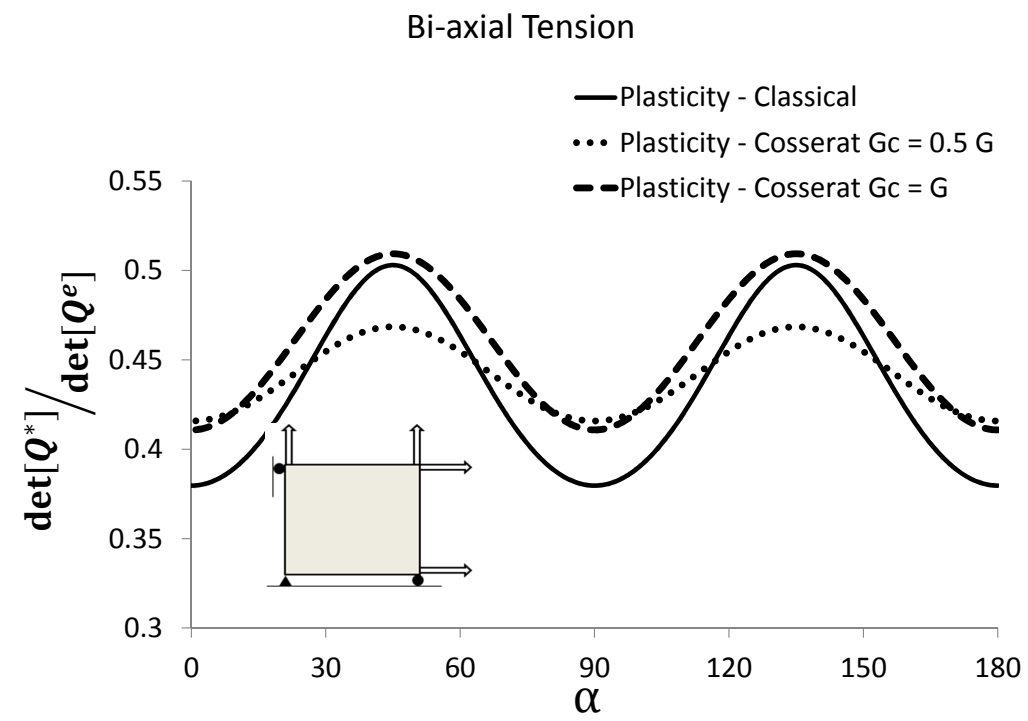

Figure 20: Comparison of the localization properties of plasticity in classical and Cosserat continua under bi-axial tension loading.

It is observed in figure 20 that although the critical angles remain the same, the Cosserat continuum enhances the overall localization properties and regularizes the localized discontinuities in the continuum. The same procedure is performed for damage-plasticity and the result is depicted in figure 21. The effect of Cosserat continuum is the same as in figure 20, however due to the effect of localized damage behavior, the graphs are shifted to the negative values. 


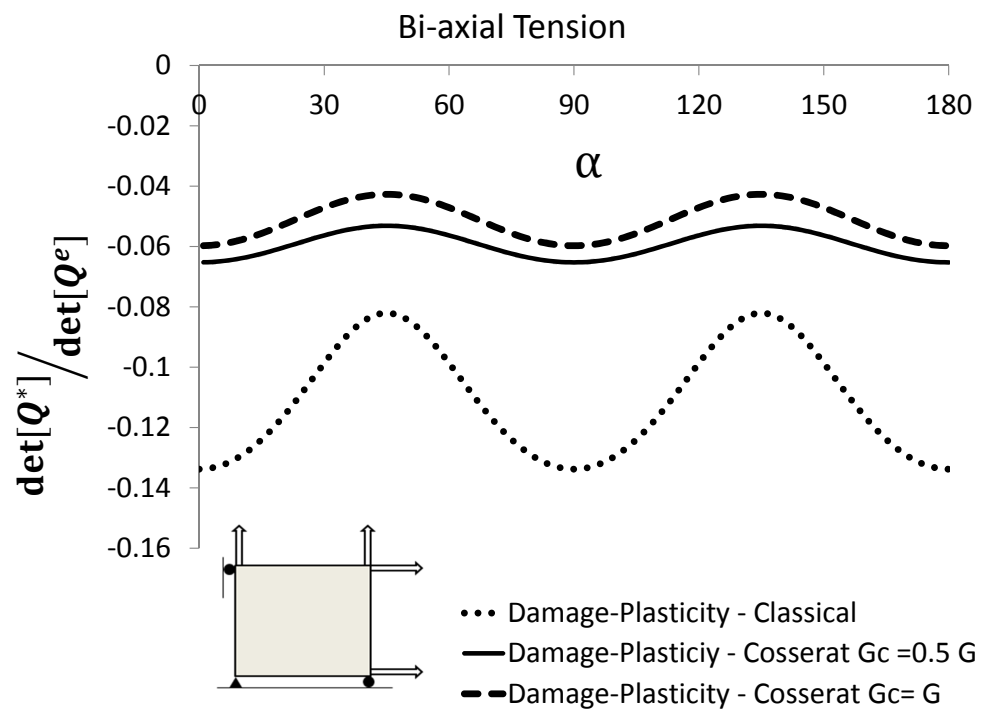

Figure 21: Comparison of the localization properties of damage-plasticity in classical and Cosserat continua under bi-axial tension loading.

\subsection{Second Localization Condition Investigations - Cosserat Continuum}

The second localization condition has been calculated for damage, plasticity and damage-plasticity formulations. Localization will only happen for Cosserat continua when the first condition is satisfied and also, due to symmetry restrictions, the rate of jump in shear stresses remains the same, i.e.

$$
\dot{\sigma}_{12}^{+}-\dot{\sigma}_{12}^{-}=\dot{\sigma}_{21}^{+}-\dot{\sigma}_{21}^{-}
$$

Assuming that $\frac{\dot{\gamma}^{\omega} \mathrm{M}_{3}^{\omega}}{\dot{\gamma}^{u}}$ is a constant, the second localization condition is solved by keeping the vector normal to the discontinuity surface $\mathbf{N}$ fixed and changing the polarization vector $\mathbf{M}$ from 0 to 360 degrees. The results are summarized in figure 22 . 


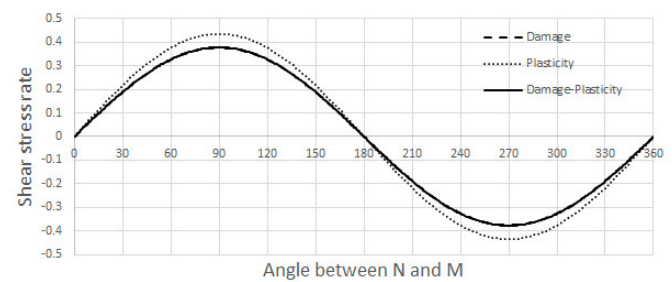

(a) Uniaxial Tension

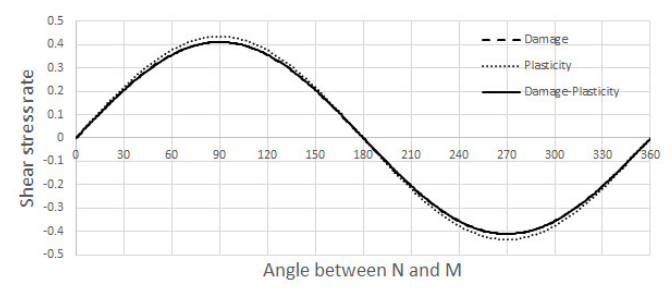

(c) Shear

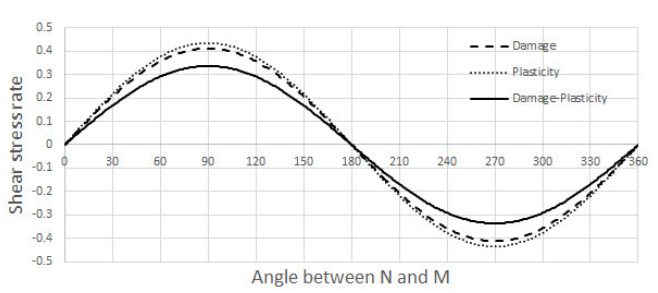

(b) Uniaxial Compression

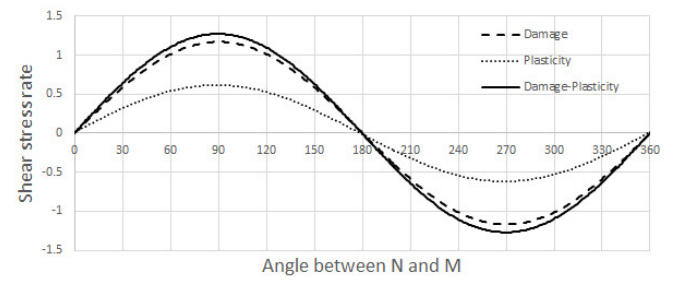

(d) Simple shear

Figure 22: Second Localization condition results for Cosserat continuum in the damage, Willam-Warnke plasticity and Damage-Plasticity cases.

As can be noticed the second localization condition for Cosserat continua is satisfied only for Mode I failure conditions. This infers that the Cosserat Damage-Plasticity model prevents localization for all Mode II and mixed mode situations when $\mathbf{N}$ is not parallel to $\mathbf{M}$ and hence provides strong regularization of failure except for Mode I [34,35].

\section{Concluding Remarks}

In this paper, the theoretical framework for modeling damage, plasticity and damage-plasticity for classical continuum is addressed. MenétreyWillam format of the Willam-Warnke plasticity surface is adopted and both hardening and softening plasticity are used to model cementitious material 
behavior. A scalar damage formulation is chosen and separate loading functions for both damage and plasticity are considered. In order to study the effect of higher order continua on the localized discontinuities, Cosserat continuum was selected and theoretical backgrounds for plasticity, damage and damage-plasticity in this formulation were discussed. Localization analysis for each of these continua were explained and the critical conditions leading to loss of ellipticity were discussed.

In the experimental observations of this paper, a series of experiments on concrete cylinders were presented and the aforementioned proposed models were calibrated according to the findings of these experiments. A digital image correlation (DIC) system was used to further investigate the strain distribution on the surface of the specimens and to capture localized failure of the samples.

The numerical localization analysis was implemented in the calibrated model and the properties of each of the damage, plasticity and damage-plasticity were examined for both classical and Cosserat continua for tension, compression, simple shear, pure shear and bi-axial tension. It was observed that plasticity formulation is more stable in comparison to damage formulation and the damage-plasticity formulation was hugely influenced by the damage formulation and therefore more susceptible to localized failure in comparison to plasticity. It was observed that reducing the formulation to von-Mises plasticity changes the critical angle of the localization and at the same time enhances the overall properties of the localization graph. Moreover the effect of the Cosserat shear modulus $G_{c}$ was studied and it was observed that by increasing this material property, the modes of failure changes and the 
susceptibility of the material to localized failure decreases. In the case of bi-axial tension it was shown that the Cosserat formulation could improve the localization properties. In the cases of shear loading, it was observed that the Cosserat continuum shows a non-symmetrical localization graph which is due to the presence of couples stresses and micro curvatures, this implies that localized failure only happens in one direction rather than having two possible directions.

As regards the second localization condition results for Cosserat continuum it was noted that this condition is satisfied only when $\mathbf{N}$ is parallel to $\mathbf{M}$ (Mode I failure); so preventing localization and providing failure regularization for Mode II and mixed mode situaitions.

In summary, the use of Cosserat continuum could reduce the localization tendencies of the plasticity and consequently the damage-plasticity formulation. The value of the Cosserat shear modulus hugely influences the localization behavior of the material and therefore could be selected in a way that regularizes the localized behaviors and improve the convergence of the finite element simulation.

\section{Acknowledgements}

The research was partially supported by the US National Science Foundation under NSF grant ID 1100971 on "Interface Mechanics of Masonry Panels under Biaxial Loading" and by the Department of Energy under NEUP grant on "Probabilistic Multi-Hazard Assessment of Dry Cask Structures". Opinions expressed are those of the authors and do not reflect those of the sponsors. 


\section{Vitae}

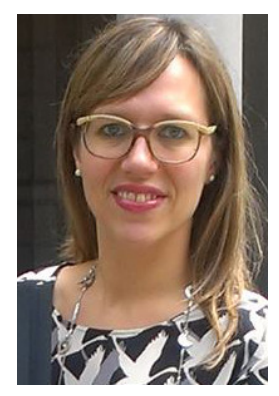

Giovanna Xotta is currently a Postdoctoral Research Fellow at the Department of Civil, Environmental and Architectural Engineering of the University of Padova. She achieved her PhD title in 2012 and worked as Postdoctoral Fellow 1 at the Department of Civil and Environmental Engineering at the University of Houston (2012-2014), under the supervision of Prof. Willam. Her main research field deals with numerical multiscale modelling of cementitious materials, considering a coupled hygro-thermo-mechanical formulation. She is currently interested on localization analysis of coupled damageplasticity models and finite element least square error analysis of digital image correlation data of deformation fields and cracks.

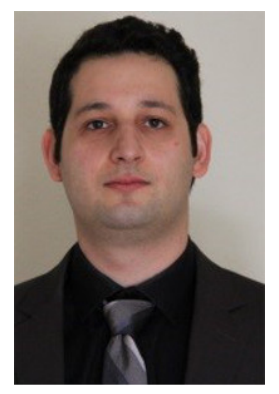

Shahriyar Beizaee received his Bachelor of Science in mechanical engineering from Iran University of Science and Technology, Tehran, Iran, in 2008, and his Master of Science in mechanical engineering from Chalmers University of Technology, Gothenburg, Sweden in 2010. He received his Ph.D. degree in civil engineering from University of Houston, Houston, Texas in 2013 and continued his research as a research fellow at the University of Houston. His current research interests include computational mechanics, plasticity, damage, fracture mechanics and failure mechanisms. 


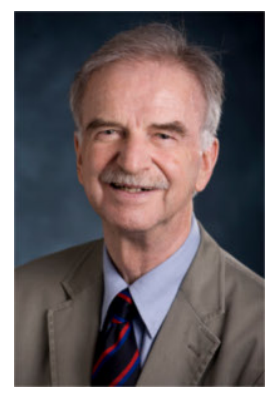

Kaspar Willam is member of the US National Academy of Engineering. His expertise is in computational failure mechanics of cohesive frictional materials. He developed a threeinvariant plasticity model known under the name of "Fiveparameter model for concrete" by Willam-Warnke. His current interests include thermo-mechanical studies of concrete spalling and finite element least square error analysis of digital image correlation data of deformation fields and cracks. Willam received his PhD degree in 1969 (University of California Berkeley) and published over 160 archival articles. Since 2010 he holds the position of Cullen Distinguished Professor of Engineering at the University of Houston.

\section{APPENDIX}

Invariants in Cosserat continuum

$$
\begin{aligned}
J_{2}=\frac{1}{2}\left(\bar{S}_{i j} \bar{S}_{i j}+\bar{m}_{i j} \bar{m}_{i j}\right)=\frac{1}{2}\left[\sigma_{x x}^{2}+\sigma_{y y}^{2}+\sigma_{z z}^{2}+\left(\sigma_{x y}+\sigma_{y x}\right)^{2}+\left(\sigma_{z x}+\sigma_{x z}\right)^{2}\right. \\
\left.+\left(\sigma_{y z}+\sigma_{z y}\right)^{2}\right]+\frac{1}{2 l_{t}^{2}}\left(m_{x x}^{2}+m_{y y}^{2}+m_{z z}^{2}\right) \\
+\frac{1}{2 l_{c}^{2}}\left(m_{y x}^{2}+m_{z x}^{2}+m_{x y}^{2}+m_{z y}^{2}+m_{x z}^{2}+m_{y z}^{2}\right)
\end{aligned}
$$


where $m$ is the couple stress matrix and is defined as

$$
\mathbf{m}=\left[\begin{array}{ccc}
m_{x x} & m_{x y} & m_{x z} \\
m_{y x} & m_{y y} & m_{y z} \\
m_{z x} & m_{z y} & m_{z z}
\end{array}\right]
$$

In two dimensional case, only $m_{x z}$ and $m_{y z}$ components are present and the rest are zero.

$$
\begin{gathered}
J_{3}=\frac{1}{3}\left(\bar{S}_{i j} \bar{S}_{j k} \bar{S}_{i k}+\bar{m}_{i j} \bar{m}_{j k} \bar{m}_{i k}\right)= \\
\frac{1}{3}\left(\sigma_{x x}^{3}+\sigma_{y y}^{3}+\sigma_{z z}^{3}\right)+\frac{1}{8}\left[\left(\sigma_{x y}+\sigma_{y x}\right)^{2}\left(\sigma_{z x}+\sigma_{x z}\right)^{2}\left(\sigma_{y z}+\sigma_{z y}\right)^{2}\right] \\
+\frac{1}{2}\left[\left(\sigma_{x y}+\sigma_{y x}\right)^{2}+\left(\sigma_{z x}+\sigma_{x z}\right)^{2}\right] \sigma_{x x}+\frac{1}{2}\left[\left(\sigma_{x y}+\sigma_{y x}\right)^{2}+\left(\sigma_{y z}+\sigma_{z y}\right)^{2}\right] \sigma_{y y} \\
\frac{1}{2}\left[\left(\sigma_{y z}+\sigma_{z y}\right)^{2}+\left(\sigma_{x z}+\sigma_{z x}\right)^{2}\right] \sigma_{z z}+\frac{1}{3 l_{t}^{3}}\left(m_{x x}^{3}+m_{y y}^{3}+m_{z z}^{3}\right) \\
\frac{m_{x x}}{3 l_{t} l_{c}^{2}}\left(m_{x y}^{2}+m_{z x}^{2}+m_{y x}^{2}+m_{z x}^{2}+m_{x y} m_{y x}+m_{z x} m_{x z}\right) \\
\frac{m_{y y}}{3 l_{t} l_{c}^{2}}\left(m_{y x}^{2}+m_{y z}^{2}+m_{x y}^{2}+m_{z y}^{2}+m_{x y} m_{y x}+m_{y z} m_{z y}\right) \\
\frac{m_{z z}}{3 l_{t} l_{c}^{2}}\left(m_{z x}^{2}+m_{z y}^{2}+m_{z x}^{2}+m_{y z}^{2}+m_{x z} m_{z x}+m_{z y} m_{y z}\right) \\
+\frac{1}{3 l_{c}^{3}}\left(m_{z y} m_{y x} m_{x z}+m_{y z} m_{z x} m_{y x}+m_{y x} m_{x z} m_{y z}+m_{x y} m_{y z} m_{x z}\right)
\end{gathered}
$$

\section{REFERENCES}

[1] J. Lee, G. Fenves, Plastic-Damage Model for Cyclic Loading of Concrete Structures, J. Eng. Mech. 124(8) (1998) 892-900.

[2] I. Carol, E. Rizzi, K. Willam, On the formulation of anisotropic elastic degradation. I. Theory based on a pseudo-logarithmic damage tensor rate, Int. J. Solids Struct. 38(4) (2001) 491-518. 
[3] M.R. Salari, S. Saeb, K.J. Willam, S.J. Patchet, R.C. Carrasco, A coupled elastoplastic damage model for geomaterials, Comput. Methods in Appl. Mech. Engrg. 193(27-29) (2004) 2625-2643.

[4] X. Tao, D.V. Phillips, A simplified isotropic damage model for concrete under bi-axial stress states, Cem. Concr. Compos. 27(6) (2005) 716-726.

[5] P. Grassl, M. Jirásek, Damage-plastic model for concrete failure, Int. J. Solids Struct. 43 (2006) 7166-7196.

[6] P. Grassl, M. Jirásek, Plastic model with nonlocal damage applied to concrete, Int. J. Numer. Anal. Meth. Geomech. 30 (2006) 71-90.

[7] J. Cěrvenka, V.K. Papanikolaou, Three dimensional combined fractureplastic material model for concrete, Int. J. Plast. 24(12) (2008) 21922220 .

[8] G.Z. Voyiadjis, Z.N. Taqieddin, P.I. Kattan, Anisotropic damageplasticity model for concrete, Int. J. Plast. 24(10) (2008) 1946-1965.

[9] P. Grassl, D. Xenos, U. Nyström, R. Rempling, K. Gyltoft, CDPM2: A damage-plasticity approach to modelling the failure of concrete, Int. J. Solids Struct. 50(24) (2013) 3805-3816.

[10] K. Willam, E.P. Warnke, Constitutive Models for the Triaxial Behavior of Concrete, Proceedings of the International Assoc. for Bridge and Structural Engineering, 19, 1-30, 1975.

[11] J.W. Rudnicki, J.R. Rice, Conditions for the Localization of Deforma- 
tion in PressureSensitive Dilatant Materials, J. Mech. Phys. Solids 23 (1975) 371-394.

[12] J.R. Rice, J.W. Rudnicki, A Note on Some Features of the Theory of Localization of Deformation, Int. J. Solids Struct. 16 (1979) 597-605.

[13] J. Hadamard, Lecons sur la Propagation des Ondes et les Equations de Hydrodynamique, Librairie Scientifique A. Hermann, Paris, France, 1903.

[14] R. Hill, Acceleration Waves in Solids, J. Mech. Phys. Solids 10 (1962) $1-16$.

[15] D. Bigoni, T. Hueckel, Uniqueness and localization - I. Associative and non-associative elastoplasticity, Int. J. Solids Struct. 28(2) (1991) 197213.

[16] D. Bigoni, T. Hueckel, Uniqueness and localization - II. Coupled elastoplasticity, Int. J. Solids Struct. 28(2) (1991) 215-224.

[17] H.D. Kang, K.J. Willam, Localization Characteristics of Triaxial Concrete Model, J. Eng. Mech. 125 (1999) 941-950.

[18] T. Liebe, K.J. Willam, Localization Properties of Generalized DruckerPrager Elastoplasticity, J. Eng. Mech. 127 (2001) 616-619.

[19] E. Rizzi, I. Carol, K. Willam, Localization Analysis of Elastic Degradation with Application to Scalar Damage, J. Eng. Mech. 121(4) (1995) 541-554. 
[20] P. Steinmann, K. Willam, Finite Element Analysis of Elastoplastic Discontinuities. J. Eng. Mech. 120 (1994) 2428-2442.

[21] S.M. Vrech, G. Etse, Geometrical localization analysis of gradientdependent parabolic DruckerPrager elastoplasticity, Int. J. Plast. 22 (2006) 943-964.

[22] K.J. Willam, M.M. Iordache, Computational Simulation of Discontinuous Failure Processes, Mecanica Computacional, 17 (1996) 227-242.

[23] K.J.Willam, M.M. Iordache, On the Lack of Symmetry in Materials, Proceedings of International Conference Trends in Computational Structural Mechanics, 233-242, 2001.

[24] V.A. Eremeyev, L.P. Lebedev, H. Altenbach, Foundations of Micropolar Mechanics, Springer Berlin Heidelberg: Berlin, Heidelberg, 2013.

[25] F. Cosserat, E. Cosserat, Sur la théorie de l'élasticité, Ann. Toulouse, 10 (1896) 1-116.

[26] F. Cosserat, E. Cosserat, Théorie des Corps Déformables, Herman, Paris, 1909.

[27] K.J. Willam, A. Dietsche, M.M. Iordache, P. Steinmann, Localization in Micropolar Continua, Chapter 9 in: H.-B. Mühlhaus (ed.), Continuum Models for Materials with Microstructure, John Wiley \& Sons Ltd., Chichester, U.K., 297-340, 1995.

[28] R. De Borst, A Simulation of Strain Localization: a Reappraisal of the Cosserat Continuum, Eng. Computation 8 (1993) 317-332. 
[29] E. Kondo, Characterizing the Evolving Internal Length Scale in Strain Localization for Cosserat Media. Ph.D. Thesis, Delft University of Technology, Delft, 2010.

[30] ARAMIS System, 2011, GOM GmbH, Braunschweig, Germany.

[31] P. Menétrey, K.J Willam, Triaxial Failure Criterion for Concrete and Its Generalization, ACI Struct. J. 92 (1995) 311-318.

[32] N. Ottosen, K. Runesson, Properties of Discontinuous Bifurcation Solutions in Elastoplasticity, Int. J. Solids Struct. 27 (1991) 401-421.

[33] Matlab, 2011, Version 7.13.0.564 (R2011b), The MathWorks Inc., Natick, Massachusetts.

[34] M. Iordache, K. Willam, Localized failure analysis in elastoplastic Cosserat continua, Computer Methods in Applied Mechanics and Engineering 151(3-4) (1998) 559-586.

[35] A. Dietsche, P. Steinmann, K. Willam, Micropolar elastoplasticity and its role in localization, International Journal of Plasticity 9(7) (1993) $813-831$ 